\title{
Influence of upstream total pressure profiles on S-duct intake flow distortion
}

\author{
Grant McLelland, ${ }^{1}$ David. G. MacManus. ${ }^{2}$ Pavlos K. Zachos ${ }^{3}$, Daniel Gil-Prieto ${ }^{4}$, Matteo Migliorini ${ }^{5}$ \\ Propulsion Engineering Centre, Cranfield University, MK43 OAL, UK
}

For some embedded engine arrangements, the nature of the inlet distortion is influenced by the boundary layer characteristics at the inlet plane of the intake. This research presents the first quantitative assessment on the influence of inlet boundary layer thickness and asymmetry on the swirl distortion at the exit of an S-shaped intake. Measurements of high spatial and temporal resolution have been acquired at the outlet plane of the S-duct using time-resolved particle image velocimetry. When boundary layer profiles typical of embedded engines are introduced, the characteristic secondary flows at the outlet plane are intensified. Overall, the peak swirl intensity increases by $40 \%$ for a boundary layer which is 7 times thicker than the reference case. The unsteady modes of the S-duct remain, although the dominant fluctuations in the flow arise at a frequency $50 \%$ lower. When the inlet boundary layer profile becomes asymmetric about the intake centerline the peak swirl events at the hub are reduced by up to $40 \%$. At the tip the peak swirl intensity increases by $29 \%$. The results demonstrate that the effects of inlet boundary layer thickness and asymmetry must be carefully considered as part of engine compatibility tests for complex intakes.

\section{Nomenclature}

$\begin{array}{llll}\mathrm{a}_{\mathrm{i}}(\mathrm{t}) & =\text { Temporal coefficient for } \mathrm{i}^{\text {th }} \text { mode in Proper Orthogonal Decomposition } & {\left[\mathrm{ms}^{-1}\right]} \\ A_{\text {in }} & =\text { S-duct inlet plane area } & {\left[\mathrm{m}^{2}\right]} \\ A_{\text {out }} & =\text { S-duct outlet plane area } & {\left[\mathrm{m}^{2}\right]} \\ \mathrm{D}_{\text {in }} & =\text { S-duct inlet plane diameter }\end{array}$

\footnotetext{
${ }^{1}$ Research Fellow, Propulsion Engineering Centre.

${ }^{2}$ Professor, Propulsion Engineering Centre, Member AIAA.

${ }^{3}$ Lecturer, Propulsion Engineering Centre, Member AIAA.

${ }^{4}$ Doctoral researcher, Propulsion Engineering Centre.

${ }^{5}$ Doctoral researcher, Propulsion Engineering Centre.
} 


\begin{tabular}{|c|c|c|c|}
\hline $\mathrm{D}_{\text {out }}$ & $=$ & S-duct outlet plane diameter & {$[\mathrm{m}]$} \\
\hline $\mathrm{f}$ & $=$ & Frequency & {$[\mathrm{Hz}]$} \\
\hline$H$ & $=$ & S-duct vertical offset between inlet and outlet plane centerlines & {$[\mathrm{m}]$} \\
\hline$H_{\text {in }}$ & $=$ & Intake height at highlight plane & {$[\mathrm{m}]$} \\
\hline $\mathrm{L}$ & $=$ & S-duct axial length between inlet and outlet planes & {$[\mathrm{m}]$} \\
\hline $\mathrm{M}_{\mathrm{in}}$ & $=$ & Mach number at inlet plane of S-duct & {$[\mathrm{m}]$} \\
\hline $\mathrm{N}_{\text {modes }}$ & $=$ & Total number of modes & \\
\hline $\mathrm{PDF}^{*}$ & $=$ & Normalised Probability Density Function & \\
\hline $\mathrm{P}_{0}$ & $=$ & Total pressure & {$[\mathrm{Pa}]$} \\
\hline $\mathrm{P}_{0, \text { ref }}$ & $=$ & Total pressure in freestream flow far from surface & {$[\mathrm{Pa}]$} \\
\hline St & $=$ & Strouhal number & {$[-]$} \\
\hline$(\mathrm{u}, \mathrm{v}, \mathrm{w})$ & $=$ & Cartesian velocity components & {$\left[\mathrm{ms}^{-1}\right]$} \\
\hline $\mathrm{V}$ & $=$ & Velocity magnitude & {$\left[\mathrm{ms}^{-1}\right]$} \\
\hline $\mathrm{V}_{\mathrm{in}}$ & $=$ & In-plane velocity & {$\left[\mathrm{ms}^{-1}\right]$} \\
\hline $\mathrm{W}_{\text {ref }}$ & $=$ & Reference velocity (area-averaged axial velocity at outlet plane of S-duct) & {$\left[\mathrm{ms}^{-1}\right]$} \\
\hline $\mathrm{W}_{\infty}$ & $=$ & Axial velocity far from surface & {$\left[\mathrm{ms}^{-1}\right]$} \\
\hline$(\mathrm{x}, \mathrm{y}, \mathrm{z})$ & $=$ & Cartesian coordinates & {$[\mathrm{m}]$} \\
\hline$\alpha$ & $=$ & Swirl angle & {$\left[{ }^{\circ}\right]$} \\
\hline$\delta$ & $=$ & Boundary layer thickness & {$[\mathrm{m}]$} \\
\hline$\Delta \mathrm{t}$ & $=$ & time delay & {$[\mathrm{s}]$} \\
\hline$\theta$ & $=$ & Distortion screen angle & {$\left[{ }^{\circ}\right]$} \\
\hline$\phi_{\mathrm{i}}$ & $=$ & $\mathrm{i}^{\text {th }}$ mode in Proper Orthogonal Decomposition & \\
\hline
\end{tabular}

\section{Operators}

$\begin{array}{lll}<.> & = & \text { Time-average } \\ \bar{r} & = & \text { Ensamble average } \\ \operatorname{std}(.) & = & \text { Standard deviation } \\ \operatorname{var}(.) & = & \text { Variance }\end{array}$




\section{I.Introduction}

Convoluted intakes remain of considerable interest for a range of current and future air vehicle configurations $[1][6][16]$. One example application is on boundary layer ingestion aircraft, which rely on partially or deeplyembedded engines to obtain gains in propulsive efficiency and reduced fuel burn [1]. However, unlike in conventional, pylon-mounted engine configurations, the engines for embedded architectures are required to operate continuously with highly-distorted intake flow. High levels of pressure and swirl distortion are present at the fan face due to the combined influence of the ingestion of the airframe boundary layer and the generation of secondary flows by the convoluted intake. The flow distortion can be highly dynamic [2][3][4][5] and has the potential to adversely influence the stability and operability of the engine [6][7][8]. Studies have demonstrated that the levels of intake flow distortion can be reduced by redesign of the intake geometry [7], or the use of flow control [9][10]. However, as the flow distortion cannot be removed completely, it is necessary to develop turbomachinery designs which are tolerant to continuous operation in distorted flow. Sufficient understanding of the characteristics of the intake flow distortion across the range of likely operating conditions is required to ensure that such distortion-tolerant designs are successful.

The nature of the secondary flows which are generated by convoluted intakes has received considerable attention in the literature. Wellborn et al. [11] demonstrated that the cross-stream pressure gradient inside the intake drives boundary layer fluid towards the inner surface of the bend and causes flow separation. As a consequence, the timeaveraged flow at the outlet plane contains a pair of counter-rotating vortices in addition to a region of total pressure loss on the lower half of the outlet plane of the duct. Zachos et al. [3] and Gil-Prieto et al. [4][12] performed extensive studies using Particle Image Velocimetry (PIV) at the outlet plane of two representative S-duct intakes. It was found that although the time-average flow field corresponds to a twin-swirl structure, the flow oscillates between positive and negative bulk swirl topologies with an intermediate condition of two contra-rotating vortices. The peak swirl distortion events were associated with the bulk swirl topologies. Modal decomposition using Proper Orthogonal Decomposition demonstrated that most of the fluctuations correspond to a swirl switching mode which is attributed to the bulk swirl topology [4]. The swirl switching mode is consistent with the Dean vortex oscillation which is observed in single-bended pipe flows [13][14]. Additional flow field unsteadiness comes from a vertical oscillation of the shear layer which is associated with the diffusive flow separation at the first bend of the S-duct [5]. 
The preceding assessments of S-duct aerodynamics are for cases where the thickness of the boundary layer at the inlet plane to the S-duct $\left(\delta_{\text {in }}\right)$ is small compared to the inlet height $\left(\mathrm{H}_{\mathrm{in}}\right)$. In such baseline studies, the total pressure profile which is associated with the boundary layer is uniformly distributed around the circumference of the duct. For example, in the study conducted by Gil-Prieto et al. [4], $\delta_{\text {in }} / \mathrm{H}_{\text {in }}$ ranged between 0.06 and 0.07 for duct inlet Mach numbers between 0.27 and 0.60. For novel aircraft architectures with embedded engines, however, the approaching boundary layer is expected to have a thickness which is comparable to the intake highlight diameter and is typically of a greater thickness on the lower surface of the S-duct inlet due to the presence of the airframe boundary layer. Computational studies for typical airframes with embedded engines have revealed that the boundary layer is expected to have a thickness to intake height ratio $\left(\delta_{\text {in }} / \mathrm{H}_{\text {in }}\right)$ of approximately 0.3 [9][15]. This number is highly dependent on the position of the intake on the airframe. For example, for a boundary layer ingesting configuration, the computational results of Giuliani and Chen [15] showed that the levels of total pressure distortion increased when the thickness of the incoming boundary layer was greater. Owens et al. [9] measured the total pressure distortion at the exit of a convoluted intake aiming to examine the effectiveness of various flow control strategies in mitigating the high distortion levels generated by the intake at transonic flight conditions. For this work, distortion fences were employed to develop an inlet boundary layer profile with shape factor around 1.50 and non-dimensional thickness in the region between $\delta_{\text {in }} / \mathrm{H}_{\text {in }}=0.30$ to 0.35 . Variation in the thickness of the approaching total pressure profile can occur due to changes in the boundary layer thickness when there is a greater length of wetted surface upstream of the highlight plane. For example, Rein and Koch [16] employed flat plate surfaces upstream of a typical embedded intake configuration. When the surface length upstream of the highlight plane was increased from $7.4 \mathrm{H}_{\text {in }}$ to $32.7 \mathrm{H}_{\mathrm{in}}$ there was a corresponding rise in $\delta / \mathrm{H}_{\text {in }}$ from 0.12 to 0.39 due to the growth of the boundary layer. The impact on the intake flow distortion was notable as there was a reduction in total pressure recovery of $2 \%$ and a $30 \%$ increase in DC60. However, there are no prior experiments which quantify the impact that such changes in $\delta / \mathrm{H}_{\text {in }}$ have on the steady and unsteady swirl distortion at the exit of the duct.

To date, studies of embedded engine configurations have predominantly been focused on cases where boundary layer profile at the inlet is symmetric about the intake symmetry plane. However, there are cases in which the azimuthal position of the total pressure loss is not symmetric about the S-duct centreline or located at the lower surface of the Sduct inlet.. For example, on typical blended wing body airframes with zero sideslip, the total pressure profile of the boundary layer at the highlight plane may have an azimuthal offset of up to $45^{\circ}$ relative to the intake symmetry plane 
due to the influence of spanwise flow on the wing upper surfaces [17]. A second scenario is for S-duct intakes during operation at large sideslip angles, in which it is possible for a flow separation to occur on the sides of the nacelle [6]. In such a case, the azimuthal location of the total pressure distortion is not aligned with the centreline axis of the Sduct [18]. A similar effect may occur for side-mounted S-duct intakes during high levels of airframe pitch angle. This asymmetry has a dramatic effect on the secondary flows inside the duct as the secondary flows inside the S-duct also become asymmetric. As a result, the time-averaged secondary flows change from the classical twin-swirl velocity pattern to an offset paired swirl topology which can have a significant impact on engine operability [6]. However, there exists no detailed measurements on the evolution of the swirl distortion levels as a function of the degree of asymmetry, and there is little understanding of the unsteadiness levels in terms of spatial or temporal distributions.

Further measurements by Guimarães et al. [19] found evidence that the total pressure distortion resulted in local separation on the rotor blades which significantly altered the flow angularity at the outlet plane of the rotor. As a result, the total pressure loss at the outlet plane increased by up to 7\% relative to the clean case. Similar conclusions were reached by Gunn et al. [20], who measured a $4.6 \%$ reduction in fan efficiency due to a $60^{\circ}$ sector of total pressure loss. The measurements revealed that the loss in efficiency is due to the fact that the rotor operates at an off-design condition around the entire annulus. Evidence of flow separation in the tip region of the rotor was observed due to the large changes in incidence as the blade transitions from distorted to clean inlet flow. A consequence of the nonuniformities in rotor outlet flow angularity is that downstream stator rows operate at off-design conditions. A further $0.7 \%$ reduction in efficiency was observed when the losses across the stator row were considered. The studies of Cousins [21] demonstrated that during a single revolution through typical flow distortion for a boundary layer ingestion configuration, the rotor blade transitions from stall to choking as it responds to the large changes in local flow incidence. The bias of the flow distortion towards the tip region is considered to be critical to fan stall inception. The simulations of Page et al. [22] support this observation as a notable loss of stability margin was demonstrated when low momentum flow was ingested in the tip region of the fan.

There are fewer studies on the influence of complex intake swirl distortion on turbomachinery performance. A loss of surge margin is anticipated when the swirl distortion is opposite to the fan rotation as there is increased incidence and loading on the rotor blades [6]. Meyer et al. [23] measured a reduction in surge margin of up to $25 \%$ for a turbojet engine when subject to a counter-rotating vortex pattern which is similar to the time-averaged flow at the outlet of an S-duct. In addition, the unsteady aerodynamic loading due to flow distortion has important implications 
for the aeromechanical response and stall margin loss of the fan blades. Lee et al. [24] studied the impact of crosswind on the performance and stability of a high by-pass ratio turbofan engine. The study showed that sufficiently high levels of cross-wind can cause severe separation at the lip of the intake which results in a severe loss of stall margin of the fan located further downstream. The same study also showed that even in cases with no lip separation a premature stall onset can appear due to incidence increase at the fan face. The aeroelastic aspects associated with such a stall in transonic fan configurations were previously described by Provenza et al. in [8] and Vahdati and Cumpsty in [25]. The impact of the unsteady distortions on the aeroelastic behaviour of fan configurations was previously studied in correlation to the rotational speed of the fan rotor expressed in engine orders where 1 engine order is the variation of a property associated with 1 full rotation of the engine fan. The presence of the distorted sector at the AIP results in a periodic excitation of the fan blades with engine order harmonics which present a risk of forced response across a range of fan rotation speeds (see Provenza et al. [8]). Previous measurements have demonstrated that although forced response margins can be increased through redesign of the blades, resonance at off-design conditions, such as flight idle, as well as flutter remain as key risks for some propulsion configurations (see Provenza et al. [8]). It is noted that these analyses have considered only the time-average flow distortion patterns. Crucially, the spectral content of the distortions must be characterized as this may superimpose key forcing frequencies at undesirable engine order excitations.

The aim of this research is to provide a detailed quantitative assessment of the influence of the approaching boundary layer characteristics on the swirl distortion downstream of a representative S-duct intake. Time-Resolved Particle Image Velocimetry is employed to measure the flow at the outlet plane of an S-duct. The purpose of the study is to provide a canonical assessment of the impact of changes in the approaching total pressure profile on the intake flow distortion produced by an S-duct. New insight on the swirl distortion is provided for S-duct aerodynamics in the presence of highlight total pressure profiles which are representative for typical embedded engine arrangements. The azimuthal position of the profile was varied to determine the impact of the asymmetric inlet boundary layer distortion on the flow distortion at the S-duct outlet plane. For the first time, the flowfield at the AIP as a function of fundamental changes in the total pressure distribution is characterized in terms of the mean and unsteady velocity components and swirl angle. Additionally, new understanding of the spectral content of the flow unsteadiness is provided, and proper orthogonal decomposition is applied to identify the key energy-containing structures. Finally, the intake swirl distortion descriptors are assessed to characterize and quantify the swirl distortion pattern. 


\section{II.Methods}

\section{A. Experimental arrangement}

The experimental investigations were performed using an S-duct rig (Figure 1). A centrifugal fan provides continuous flow though the rig, which comprises a bell-mouth intake of inner diameter $200 \mathrm{~mm}$ followed by flow conditioning honeycomb screens and a contraction which reduces the inner diameter to $D_{\text {in }}=121.6$ mm. A tubular section of length $2.55 \mathrm{D}_{\text {in }}$ is located upstream of the S-duct inlet plane. The S-duct geometry corresponds to the highoffset diffusing S-duct which has been investigated in previous measurements of Zachos et al.[3][6] and Gil-Prieto et al.[4][5]. The duct features an offset $\left(H / \mathrm{D}_{\text {in }}\right)$ of 2.44 , a length ratio $\left(\mathrm{L} / \mathrm{D}_{\text {in }}\right)$ of 4.95 and an overall diffusion area ratio $\left(\mathrm{A}_{\text {out }} / \mathrm{A}_{\text {in }}\right)$ of 1.52. A cylindrical, transparent working section of inner diameter $150 \mathrm{~mm}$ is located downstream of the S-duct outlet plane to facilitate PIV measurements.

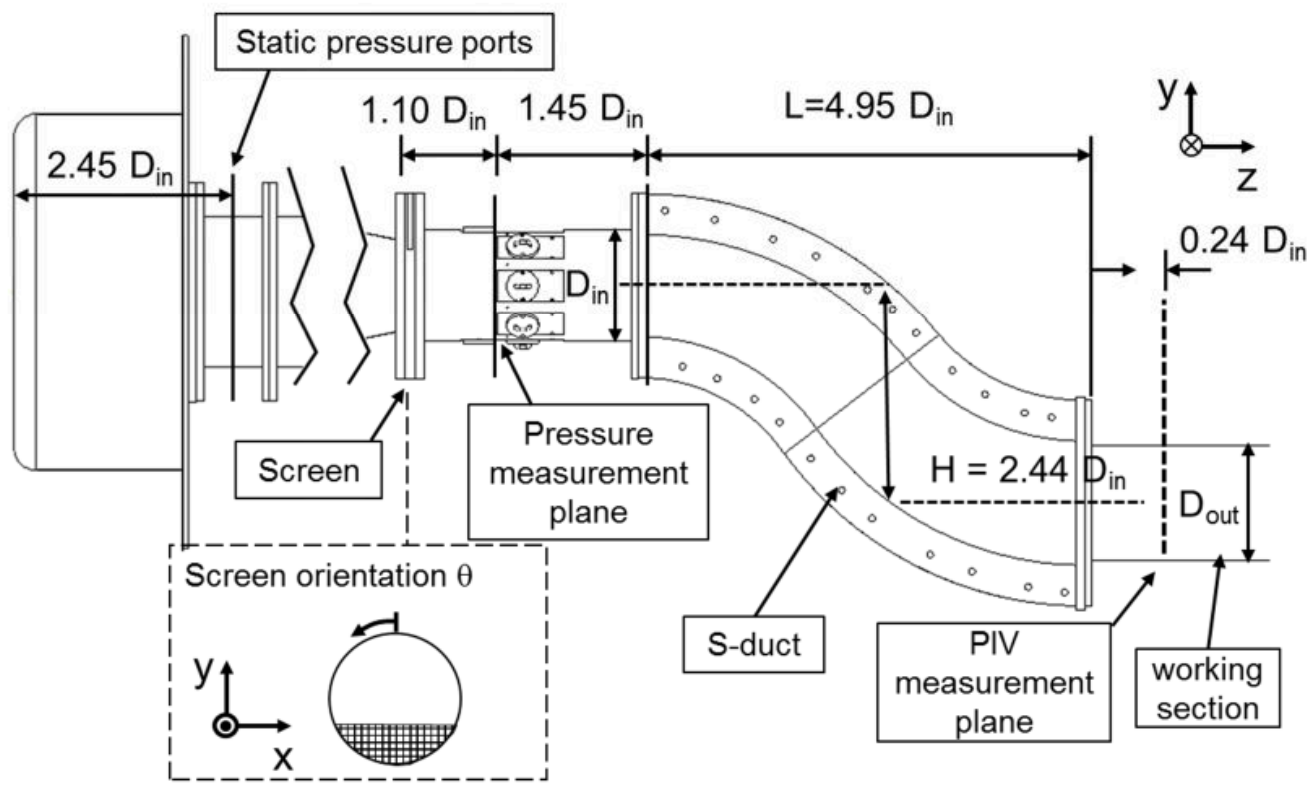

Figure 1: Schematic of experimental arrangement

\section{B. Pressure and temperature instrumentation}

To establish the operating condition of the intake flow, the static pressure was measured as a pneumatic average from 12 static pressure ports which are equi-spaced around the inner diameter of the bellmouth inlet at a distance of 
$2.45 \mathrm{D}_{\text {in }}$ downstream of bellmouth's highlight plane (Figure 1). A differential pressure transducer of range $3.7 \mathrm{kPa}$ and accuracy $0.073 \%$ full-scale is used to measure the static pressure at a frequency of $500 \mathrm{~Hz}$ and a rolling average over 1s. The ambient pressure was measured using a transducer with an accuracy of $0.01 \%$ full scale. The inlet Mach number was consequently measured with an uncertainty of $+/-0.01$ at $\mathrm{M}_{\mathrm{in}}=0.27$.

A measurement rake comprising 10 Pitot probes was placed at $1.45 \mathrm{D}_{\text {in }}$ upstream of the $\mathrm{S}$-duct inlet to measure the total pressure distribution of the approaching boundary layer with a spatial resolution of $0.06 \mathrm{D}_{\text {in }}$. Additionally, static pressure measurements were acquired at 8 static pressure ports which were located at $1.45 \mathrm{D}_{\text {in }}$ and were equi-spaced around the duct inner surface. All pressure data from the rake and static pressure ports in the calibration section were measured using a +/- $34.5 \mathrm{kPa}$ differential transducer with full-scale accuracy $0.06 \%$. The data were acquired at a frequency of $500 \mathrm{~Hz}$ for $12 \mathrm{~s}$.

\section{Time-Resolved PIV system}

Time-Resolved PIV was used to measure the three-component velocity field at the AIP, which is defined as a plane normal to the duct axis and at a distance of $0.24 \mathrm{D}_{\text {in }}$ downstream of the outlet plane of the S-duct (Figure 1). A 100W dual-cavity laser was used to provide a light sheet with an approximate thickness in the z-axis of $1.5 \mathrm{~mm}$. Two cameras with a sensor resolution of $1280 \times 800 \mathrm{px}^{2}$ were used with a camera separation half-angle of $45^{\circ}$. Lenses of focal length $28 \mathrm{~mm}$ were used along with Scheimpflug mounts to ensure adequate focus across the entire region of interest. Spatial calibration was conducted using a multi-plane traverse in the z-direction with 3 planes on an axial spacing of $0.75 \mathrm{~mm}$. Seeding particles of nominal diameter $1 \mu \mathrm{m}$ were generated using Di-Ethly-Hexyl-Sebacat oil with Laskin nozzle atomizers. The seeding was introduced into the capture streamtube of the intake by means of a seeding chamber which encloses the bell-mouth intake.

The PIV time delay $(\Delta \mathrm{t})$ was set to ensure that particle displacements in the z-axis were no greater than $1 / 4$ of the thickness of the laser light sheet, and no greater than 1/4 of the typical interrogation window dimension. The temporal measurement rate of the time resolved PIV measurements was established to ensure sufficient resolution of the key unsteady fluctuations inside the duct. Previous experimental and computational studies for the same intake geometry and operating conditions [5][12] revealed that the dominant flow fluctuations are found for Strouhal numbers $(\mathrm{St}=\mathrm{f}$ $\mathrm{D}_{\text {out }} / \mathrm{W}_{\text {ref }}$ ) of up to 1.0 , where $\mathrm{W}_{\text {ref }}$ is the average out of plane velocity at the AIP. Therefore, the key flowfield oscillations are expected to occur at frequencies below $410 \mathrm{~Hz}$ for an inlet Mach number of $\mathrm{M}_{\mathrm{in}}=0.27$ and an outlet duct diameter of $D_{\text {out }}=150 \mathrm{~mm}$. It was decided that the acquisition frequency should be 10 times as great as the 
dominant flow fluctuations. As a result, a measurement frequency of $4 \mathrm{kHz}$ was employed. A total of 20,000 snapshots of three-component velocity measurements were acquired at the AIP outlet of the S-duct.

\section{PIV data reduction}

The PIV processing included the application of a geometric mask to the raw images to mitigate the influence of laser light reflections on the working section surfaces and to ensure that only data which is contained inside the working section is utilized in the PIV processing. A background subtraction scheme was utilized to subtract the average intensity of the 20,000 images acquired for each dataset. The pre-processed images were then processed using a GPU direct correlation algorithm. An iterative multi-grid approach with a window overlap of 50\% was employed with an initial pass of $128 \mathrm{px}$ which reduced to $32 \mathrm{px}$ on the final pass. Local validation was performed using a median filter on a stencil size of $3 \times 3$ and a tolerance of 2 standard deviations. A smoothing kernel with stencil size $3 \times 3$ was applied during multi-pass and final post-processing steps. The processing strategy resulted in a final vector resolution of $2.8 \mathrm{~mm}\left(0.019 \mathrm{D}_{\text {out }}\right)$ and a total of approximately 2000 vectors across the AIP per snapshot. An iterative disparity correction was applied to mitigate uncertainties due to misalignment between the calibration plane and the laser light sheet as described by Wieneke in [26]. The overall uncertainty on the velocity components has been estimated based on the sensitivities reported by Raffel et al. [27]. Uncertainty contributions have been considered from the effects of particle image diameter, particle image density, image quantization levels, displacement gradients, and background noise. The uncertainty for the in-plane and out-of-plane velocities are estimated as $4.9 \%$ and $7.0 \%$ of the average out of plane velocity at the AIP [12]. These uncertainty figures account for no particle tracking errors due to centrifugal forces caused by the flow to the particles. As shown by Birch and Martin [28] and Raffel et al. [27], the in-plane circumferential velocity component is far less susceptible to particle momentum errors than the radial velocity. Application of Zhou's method in the current data [29], showed that the induced radial velocity to the seeding particles from the flow vortices is at least one order of magnitude less than the measured in-plane radial and circumferential velocities. Hence, the influence of the particle momentum effects on the overall velocity uncertainty is negligible and was not accounted for in the overall calculations.

\section{E. Distortion screens and test matrix}


No distortion screen was present in the baseline reference configuration. With an inlet Mach number of 0.27 this provided an inlet boundary layer thickness of $\delta_{\mathrm{in}} / \mathrm{D}_{\mathrm{in}}=0.07$ along the whole circumference based on previous measurements [4] taken at the pressure measurement plane (Figure 1). Mesh-based distortion elements were employed to create profiles of total pressure which are representative of an approaching boundary layer. Studies of typical boundary layer ingestion airframe configurations reveal that the thickness of the boundary layer at the inlet plane of a rear-mounted intake is expected to be approximately $0.3 \mathrm{H}_{\text {in }}$ [7], but may reach as much as $0.6 \mathrm{H}_{\text {in }}$ depending on the engine placement [15]. This is of significance as the distributions of total pressure loss at the AIP are sensitive to the thickness of the approaching boundary layer [15].

A representative inlet total pressure profile was selected from the measurements from Giuliani and Chen [15] to guide the design of suitable distortion screens. Giuliani and Chen's measurements were acquired at the highlight plane of a semi-circular intake with a width to height ratio of 0.95 at a freestream Mach number of 0.4 and an intake Reynolds number of $7.3 \times 10^{6}$ based on the height of the intake at the highlight plane, $\mathrm{H}_{\mathrm{in}}$. For this case, the approaching boundary layer had a thickness of $\delta_{\text {in }}=0.33 \mathrm{H}_{\text {in }}$ at the highlight plane, where $\delta_{\text {in }}$ corresponds to the location at which the isentropic flow velocity reaches $0.99 \mathrm{~W}_{\infty}$. This would also correspond to an equivalent aircraft fuselage Reynolds number per unit length of $\operatorname{Re}_{x} / 1$ of about $0.9 \times 10^{9}$ and it is representative of a full scale BWB aircraft, which is expected to have a boundary layer near the wind-body trailing edge of about 30\% [30] . In the current study, an inlet total pressure profile with $\delta_{\text {in }}=0.33 \mathrm{D}_{\text {in }}$ was generated via a distortion screen to match the Giuliani and Chen data where $\mathrm{D}_{\text {in }}$ indicates the S-duct inlet diameter (Figure 2). To quantify the influence of the approaching boundary layer thickness on the flow distortion at the AIP, an additional distortion screen was developed to increase the thickness of the approaching total pressure profile to approximately $\delta_{\text {in }}=0.51 \mathrm{D}_{\text {in }}$ (Figure 2$)$. A summary of the main properties of the profiles is reported in Table 1.

Table 1: Inlet total pressure profile characteristics

\begin{tabular}{|l|c|c|c|}
\hline & $\boldsymbol{\delta}_{\text {in }}=\mathbf{0 . 0 7}_{\text {in }}$ & $\boldsymbol{\delta}_{\text {in }}=\mathbf{0 . 3 3}_{\text {in }}$ & $\boldsymbol{\delta}_{\text {in }}=\mathbf{0 . 5 1}_{\text {in }}$ \\
\hline Displacement thickness $\left(\delta^{*} / \mathrm{D}_{\text {in }}\right)$ & 0.006 & 0.085 & 0.140 \\
\hline Reynolds number $\left(R e_{\delta^{*}}\right)$ & $4.6 \times 10^{3}$ & $6.3 \times 10^{4}$ & $1.0 \times 10^{5}$ \\
\hline
\end{tabular}

For the cases with distortion screens, the Mach number at the edge of the boundary layer $\left(y=\delta_{\text {in }}\right)$ was set to the same value as the nominal Mach number for the reference case $\left(\mathrm{M}_{\mathrm{in}}=0.27\right)$. The distortion screens were mounted $2.55 \mathrm{D}_{\text {in }}$ upstream of the inlet plane of the S-duct (Figure 1). The mount for the screen permitted the rotation of the 
screen about the tunnel z-axis to investigate different azimuthal positions. Screen angles $(\theta)$ of between $0^{\circ}$ and $90^{\circ}$ were investigated in $15^{\circ}$ increments. The screens increased the thickness of the inlet total pressure profile only in the annular portion in which the mesh is present. The remaining part of the circumference is expected to maintain the same boundary layer thickness as the baseline case $\left(\delta_{\text {in }}=0.07 \mathrm{D}_{\text {in }}\right)$.

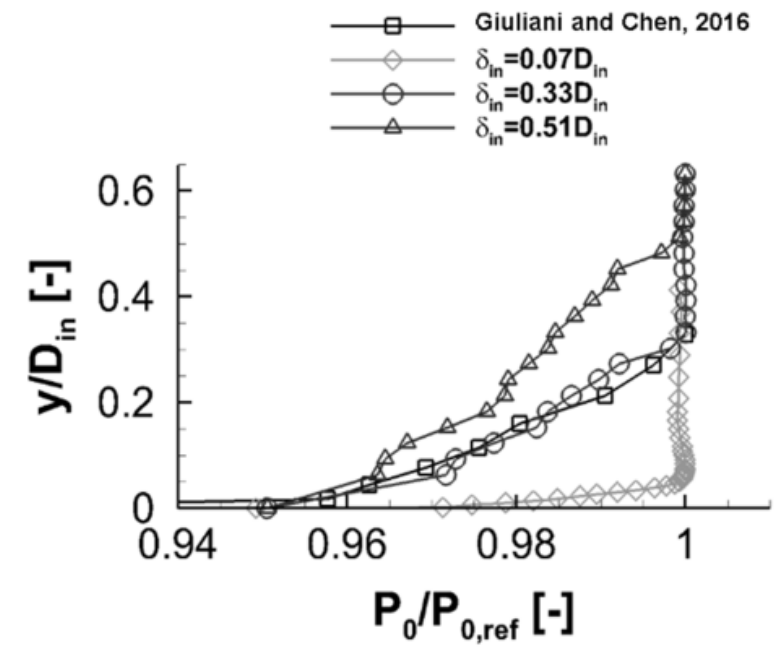

Figure 2: S-duct inlet total pressure profiles and comparison with Giuliani and Chen [15]. 


\section{III.Results and Discussion}

\section{A. Time-averaged flow field}

The time-averaged velocity and swirl angle distributions for the baseline case with $\delta_{\text {in }}=0.07 \mathrm{D}_{\text {in }}$ (Figure 3 ) exhibit the expected time-averaged twin swirl flow downstream of a typical S-duct. The flow is characterized by the presence of a large region of axial velocity deficit near the centre of the AIP and with a minimum out of plane velocity $(\langle w\rangle)$ of $0.87 \mathrm{~W}_{\text {ref, }}$ where $\mathrm{W}_{\text {ref }}$ is the time-averaged, area-averaged out of plane velocity at the AIP. The large levels of axial velocity deficit originate from the flow separation due to the adverse pressure gradient which forms just downstream of the first bend inside the S-duct [5][11]. High-momentum flow with a maximum of $\langle\mathrm{w}\rangle=1.25 \mathrm{~W}_{\text {ref }}$ is located towards the upper surface. The in-plane flow features the classical counter-rotating vortices in the lower half of the AIP and within the spoiled region (Figure 3). The time-averaged swirl angle $(\langle\alpha\rangle)$ reaches a maximum of $5.7^{\circ}$ close to the lower surface. The distribution and levels of the in-plane and out-of-plane flows are consistent with prior measurements $[3][4][5][10][12]$ on the same configuration at the same operating conditions.

When the thickness of the approaching boundary layer is increased from $\delta_{\text {in }}=0.07 \mathrm{D}_{\text {in }}$ to $0.33 \mathrm{D}_{\text {in }}$ there is a corresponding intensification of the spoiled region and the in-plane flows. For $\delta_{\mathrm{in}}=0.33 \mathrm{D}_{\mathrm{in}}$, the minimum local value of axial velocity decreases from $0.87 \mathrm{~W}_{\text {ref }}$ to $0.67 \mathrm{~W}_{\text {ref }}$ (Figure 3 ). As a consequence of the increased blockage caused by the spoiled region, the peak axial velocity increases from $1.25 \mathrm{~W}_{\text {ref }}$ to $1.37 \mathrm{~W}_{\text {ref }}$ (Figure 3). The increased inlet boundary layer thickness also amplifies the secondary flows at the AIP. As a result, the local peak swirl angle rises notably from $5.7^{\circ}$ to $14.2^{\circ}$ (Figure 3 ). The overall characteristics of the flow distortion are comparable to those measured by Giuliani and Chen [15] at the same $\delta_{\text {in }} / D_{\text {in }}$, in which the lower portion of the S-duct outlet plane featured a region of spoiled flow with counter-rotating vortices. Results expressed in the rotor frame of reference revealed that the flow distortion increased the relative incidence angle by up to approximately $10^{\circ}$ in the outer annuli of the duct at the fan face plane. A similar test case [31] showed that absolute swirl angles could increase up to $10^{\circ}$ in the main coswirling and counter-swirling regions. Prior studies [3][4] have demonstrated that the peak swirl angles at the S-duct outlet are proportional to the duct offset ratio (h/Din). Therefore, the higher values of $\langle\alpha\rangle$ as reported in the current research $\left(\langle\alpha\rangle=14.2^{\circ}\right)$ are expected due to the larger value of duct offset $(\mathrm{H} / \mathrm{Din}=2.44)$, compared to $\mathrm{H} / \mathrm{Din}=1.49$ in [15]. Moreover, the unsteady measurements are expected to depict peak swirl events which have a magnitude greater than time-averaged ones [12]. Further increases in the intensity of the spoiled region and the secondary flows are 
observed for $\delta_{\text {in }}=0.51 D_{\text {in }}$ (Figure 3 ). The maximum swirl angle reaches $\langle\alpha\rangle=16.3^{\circ}$ which is almost three times the magnitude of $\langle\alpha\rangle$ which was observed for the baseline case $\left(\delta_{\mathrm{in}}=0.07 \mathrm{D}_{\mathrm{in}}\right)$. This dependence of the flow distortion on $\delta_{\text {in }} / \mathrm{D}_{\text {in }}$ is consistent with the trends which were reported in Rein and Koch [16], in which in the distributions of total pressure at the S-duct outlet also showed a deepening of the intensity of the spoiled region with increases in $\delta_{\text {in }} / D_{\text {in }}$.

Previous investigations using the same geometry as the current study revealed high levels of flow unsteadiness due to the combination of the velocity fluctuations attributed to the diffusion led separation and the unsteady oscillations of the streamwise vortices at the AIP [3][4][5]. The baseline case $\left(\delta_{\text {in }}=0.07 \mathrm{D}_{\text {in }}\right)$ is in agreement with these previous measurements and features notable levels of unsteadiness on all velocity components (Figure 4). The unsteadiness is primarily contained in the lower half of the AIP, and the standard deviation values reach a local maximum of $0.23 \mathrm{~W}_{\text {ref }}$ for the $\mathrm{u}$-component of velocity. The peak unsteadiness in swirl angle of std( $\left.\alpha\right)$ of $14.5^{\circ}($ Figure 4) is 2.5 larger than the time-averaged value and remains located in the lower half of the AIP. As the boundary layer thickness $\delta_{\text {in }}$ is increased, there is a progressive increase in the intensity and the extents of the flow field unsteady region. For example, the local peak unsteadiness in swirl angle reaches $\operatorname{std}(\alpha)=22.5^{\circ}$ for $\delta_{\text {in }}=0.51 D_{\text {in }}($ Figure 4$)$ which is four times greater than the peak time-average reference case $\left(\delta_{\mathrm{in}}=0.07 \mathrm{D}_{\mathrm{in}}\right)$.

The overall increase in flow non-uniformity and unsteadiness is consistent with an intensification of the diffusive separation inside the duct. This is because the thicker boundary layer on the lower surface of the inlet plane is expected to be more susceptible to separation under the adverse pressure gradients which occur after the first turn in the duct.

The effect of rotating the inlet total pressure profile around the z-axis on the steady and unsteady flow field at the AIP was also assessed. Prior investigations [6] have revealed that the secondary flows which are generated at the outlet of an S-duct become asymmetric when the approaching boundary layer is no longer symmetric about the centerline of the duct. The measurements in this study confirm that the azimuthal position of the incoming total pressure deficit has a first-order effect on the time-averaged flow topology at the AIP (Figure 5). As the screen angle ( $\theta$ ) increases from $0^{\circ}$, there is a progressive transition from a paired-swirl topology to one in which the clockwiserotating vortex becomes increasingly dominant. This establishes negative swirl angles in the tip region and introduces an additional region of positive swirl angles close to the hub. The transition to a flow field with a dominant clockwise swirl is more rapid when $\delta_{\text {in }}$ is increased to $0.51 \mathrm{D}_{\text {in }}$ (Figure 6). The greatest level of time-averaged swirl is observed for $\theta=90^{\circ}$ with a maximum of $|\langle\alpha\rangle|_{\max }=28.0^{\circ}$ (Figure 6). An important consequence is that changes in the orientation 
and thickness of the inlet boundary layer profile result in time-averaged swirl angles in the tip region of a fan which are approximately two times as large as those for an axisymmetric inlet boundary layer with a small thickness compared to the intake inlet diameter.

Overall, the unsteadiness in the flow field reduces as the screen angle is increased (Figure 7), such that the peak $\operatorname{std}(\alpha)$ for $\delta_{\text {in }}=0.33 \mathrm{D}_{\text {in }}$ reduces from $19.8^{\circ}$ at $\theta=0^{\circ}$ to $14.5^{\circ}$ at $\theta=90^{\circ}$ (Figure 8). Similar trends are observed for $\delta_{\text {in }}=0.51 \mathrm{D}_{\text {in. }}$. Therefore, it is evident that an asymmetric inlet boundary layer profile is beneficial in terms of overall unsteadiness in the velocity components when compared to the symmetric case. However, this result should be considered in conjunction with the assessment of the swirl distortion descriptors (section D) as it is the peak absolute values of unsteady swirl distortion which is of primary interest from the perspective of the turbomachinery response.

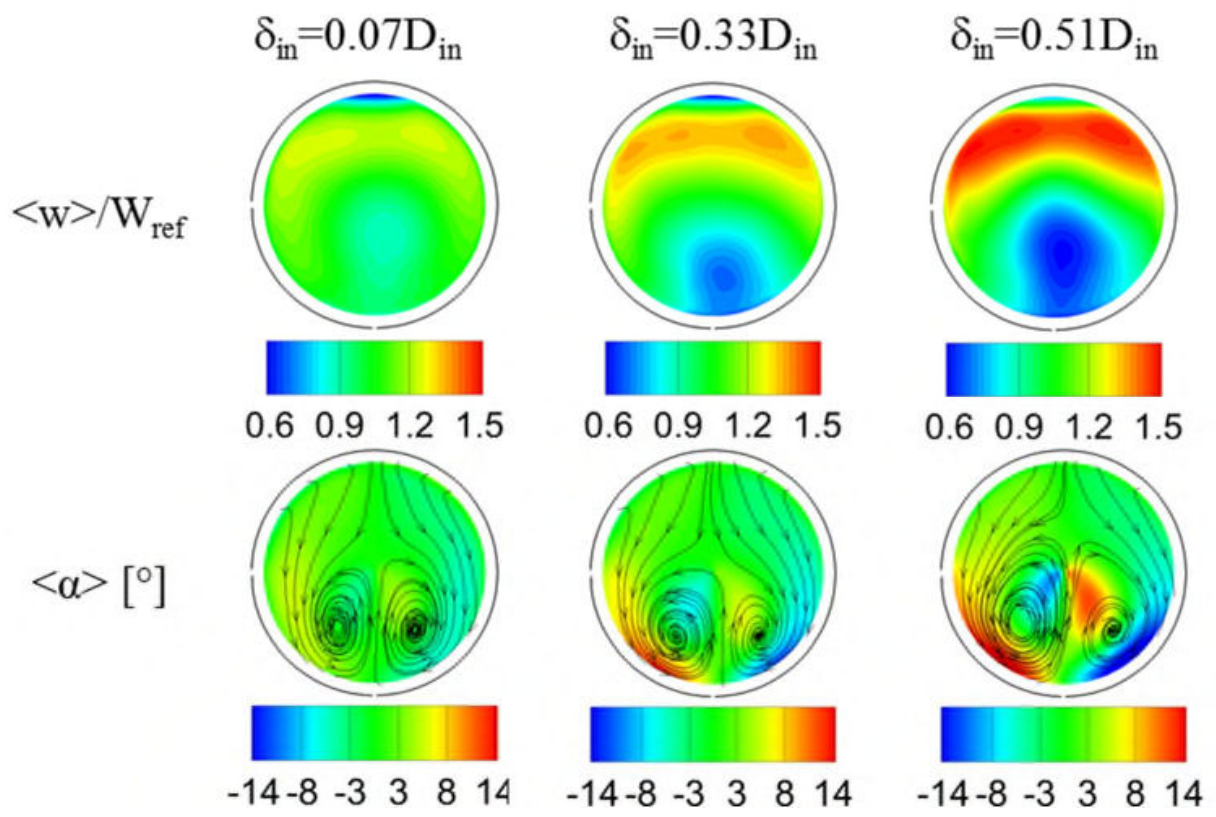

Figure 3: Normalised time-averaged streamwise velocity $\left(\langle w\rangle / W_{\text {ref }}\right)$ and swirl angle $(\langle\alpha\rangle)$ for $\delta_{\text {in }}=0.07 D_{\text {in }}, 0.33 D_{\text {in }}$ and $0.51 D_{\text {in }}$ at $\theta=0$. 


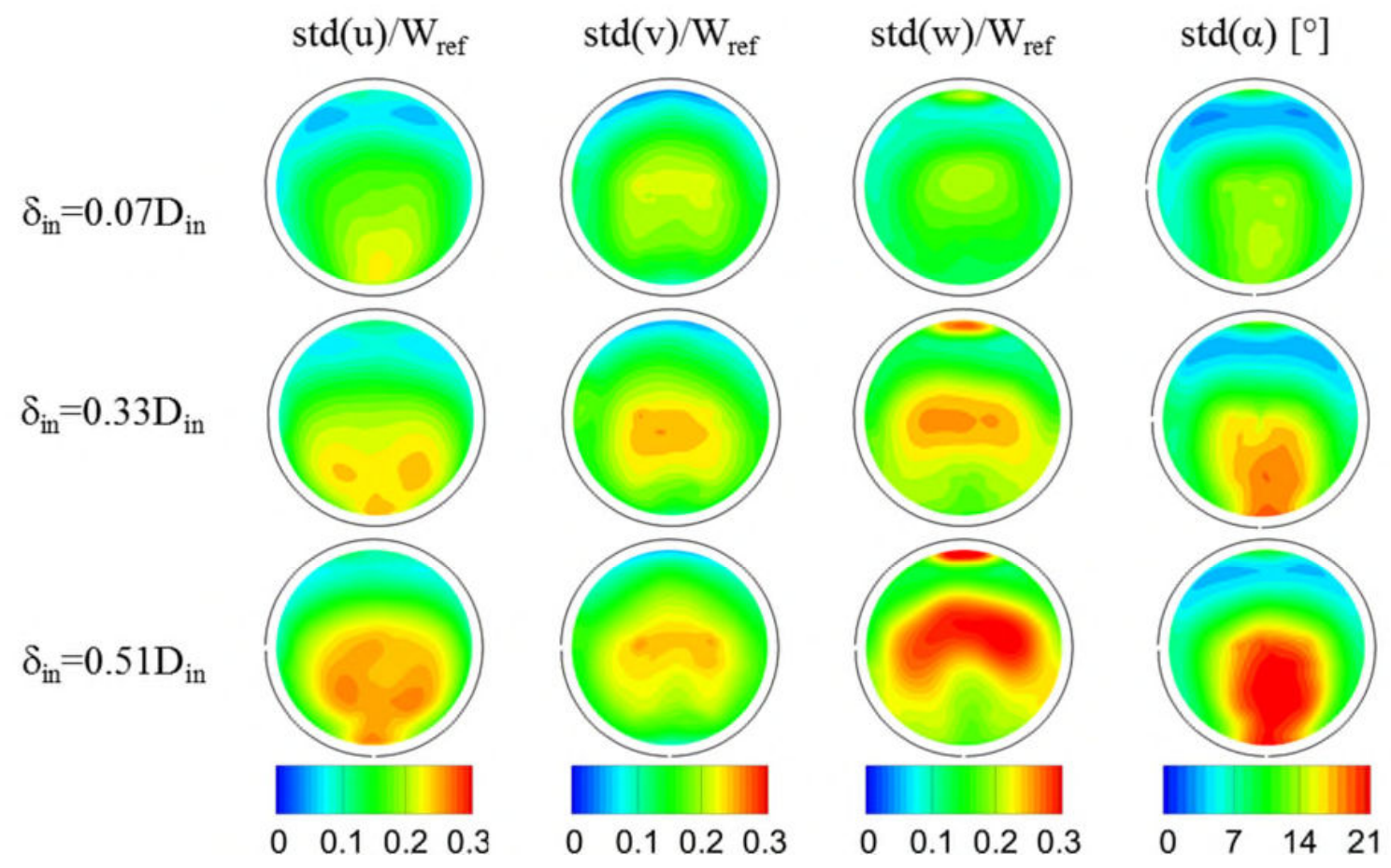

Figure 4: Unsteady velocity $[\operatorname{std}(u), \operatorname{std}(v), \operatorname{std}(w)] / W_{\text {ref }}$ and swirl angle $(\operatorname{std}(\alpha))$ for $\delta_{\text {in }}=0.07 D_{\text {in }}, 0.33 D_{\text {in }}$ and $0^{.51 D} D_{\text {in }}$ at $\theta=0$.

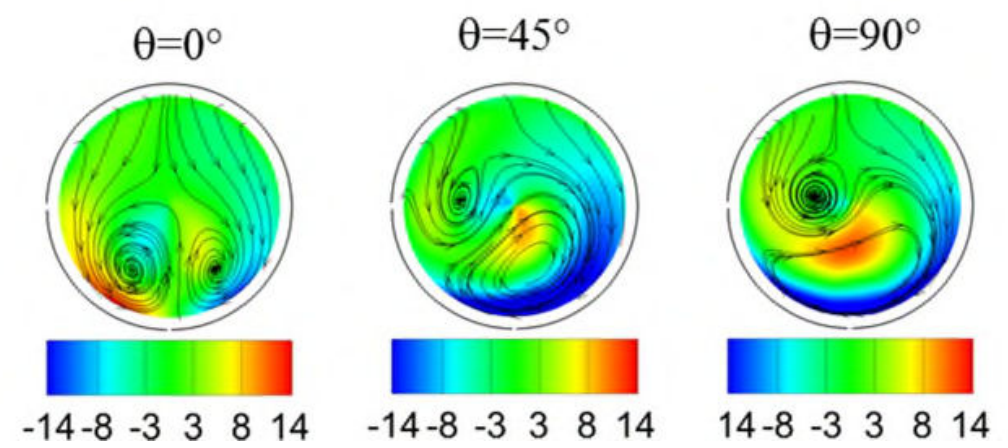

Figure 5: Effect of the screen angle on the time-averaged swirl angle $(\langle\alpha\rangle)$ at $M_{i n}=0.27$, for the flow profile with $\delta_{\text {in }}=0.33 D_{\text {in }}$ 


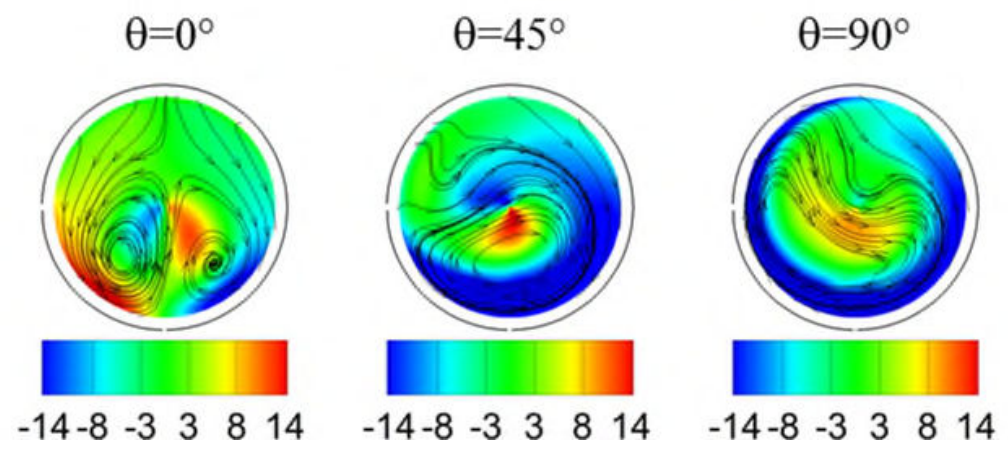

Figure 6: Effect of the screen angle on the time-averaged swirl angle $(\langle\alpha\rangle)$ at $M_{\text {in }}=0.27$, for the flow profile with $\delta_{\text {in }}=0.51 D_{\text {in }}$.

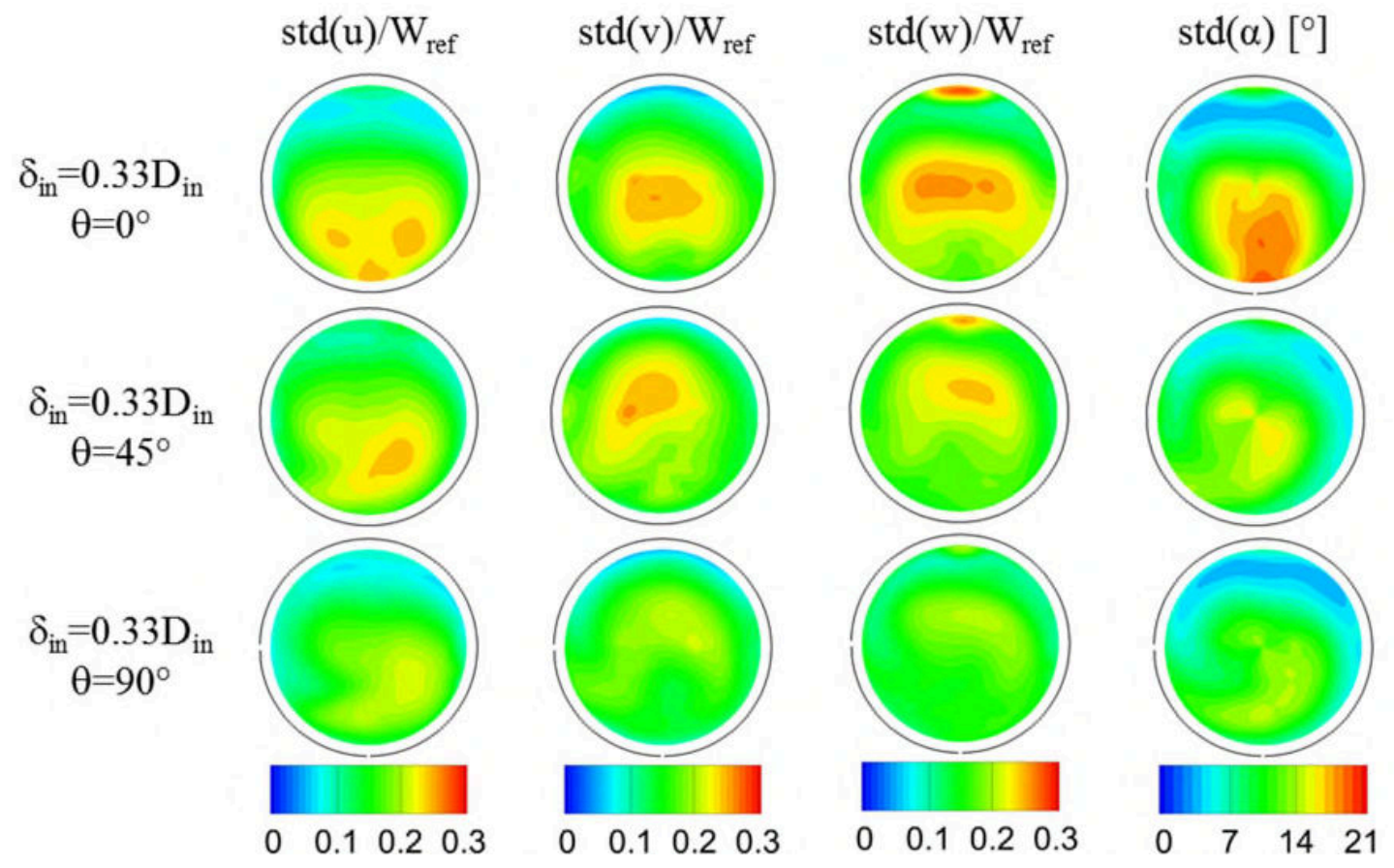

Figure 7: Unsteady velocity components $[\operatorname{std}(u), \operatorname{std}(v), \operatorname{std}(w)] / W_{\text {ref }}$ and swirl angle $(\operatorname{std}(\alpha))$ for $\delta_{\text {in }}=0.33 D_{\text {in }}$ as a function of $\theta$ 


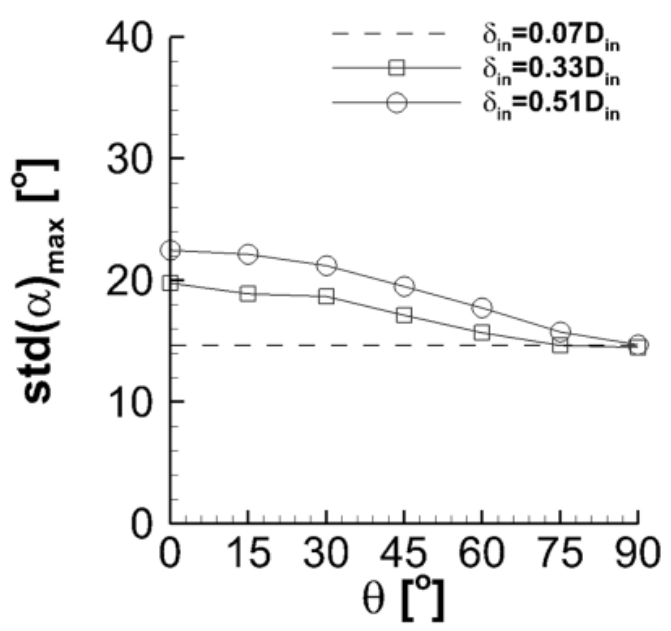

Figure 8: Maximum value of $\operatorname{std}(\alpha)$ at $\mathrm{AIP}$ as a function of screen angle $\theta$

\section{B. Flow field spectral analysis}

The frequency of the unsteady flow fluctuations is known to be an important parameter for the response of a fan as it requires a finite amount of time to respond to changes in the intake flow conditions [32]. There is very little open source information on the likely response of a compression system to unsteady swirl distortion.

For total pressure distortions, it is recognized that the unsteady characteristics of flow distortion have an influence on the response of a compressor when the unsteadiness lies within a particular band of frequencies [32]. When the unsteadiness occurs at very high frequencies, the changes in flow distortion are too rapid for the compressor to respond. Cousins [21] states that the time which is taken for a distortion to convect from the leading edge to the throat of the rotor blade is important in defining the response of the compressor to distortion. However, a typical assumption which is made is that distortions which persists for a duration which is less than the equivalent time for $60^{\circ}$ of the rotor revolution are expected to have a negligible impact on fan stability [22]. Similarly, for sufficiently slow variations in intake distortion, the response of the fan is primarily influenced by the spatial distribution of the steady-state flow distortion. It is considered that the compressor response is relatively benign for pressure distortions which occur at frequencies of less than one engine order [33].

For example, for Rotor 67 [34] operating at design rotational speed, the maximum Strouhal number at which a response is expected, as defined by the equivalent duration for a $60^{\circ}$ rotor revolution, is for $\mathrm{St}=\left(\mathrm{f} \mathrm{D}_{\text {out }} / \mathrm{W}_{\text {ref }}\right)=4.9$. This is notably lower than the frequency based on the leading edge to throat convection which corresponds to St $=$ 
11.6. It is assumed that flow field unsteadiness with St of less than 4.9 are in the range which may provoke an unsteady aerodynamic response. Similarly, frequencies of less than one engine order $\left(\mathrm{St}_{1 \mathrm{EO}}=0.8\right)$ define a lower limit for unsteady response. Therefore, based on guidance for total pressure distortions, for this example the key region of interest for flow field unsteadiness in terms of unsteady aerodynamic response corresponds to $0.8<\mathrm{St}<4.9$. Additional guidance on the key frequencies of interest can be obtained from the perspective of aeromechanical response. For a typical boundary layer ingestion rotor, the first four resonant modes occur for Strouhal numbers of between 0.7 and 3.8 [8]. Flow unsteadiness within this range of Strouhal numbers has the potential to generate a forced response on the blades. Consequently, overall an indicative range of interest for the measurements is $0.7<\mathrm{St}<4.9$.

The spectral content of the variance of the velocity magnitude has been calculated using the average periodogram method [35]. The power spectrum for each dataset has been computed as the ensemble average of the power spectra which were evaluated from 20 segments of 1000 snapshots. Hanning windowing was applied to mitigate spectral leakage. The processing strategy has resulted in a frequency resolution of $\Delta \mathrm{St}=0.01$, with a maximum resolvable frequency of $\mathrm{St}=5.0$. The power spectrum has been subdivided into discrete spectral bands with step size $\delta \mathrm{St}=0.2$ to determine the contribution of each band to the overall variance.

For the reference case of $\delta_{\text {in }}=0.07 \mathrm{D}_{\text {in }}$, the flow unsteadiness is distributed across a range of bands from $\mathrm{St}=0.0$ to 1.0. For St $>1.0$ the contribution to the overall unsteadiness becomes negligible, in agreement with previous measurements [12]. The greatest contribution to the flow field unsteadiness is located within a spectral band range of $\mathrm{St}=0.4$ to 0.6 (Figure 9) which is close to the lower limit on the range of frequencies of interest $(\mathrm{St}=0.7)$. Therefore, the fluctuations in the flow field have the potential to induce unsteady aerodynamic and aeromechanical effects on a typical rotor.

When the boundary layer thickness is increased to $\delta_{\mathrm{in}}=0.33 \mathrm{D}_{\mathrm{in}}$, the spatial distributions of unsteadiness remain qualitatively similar to the baseline case, but there is an overall increase in unsteadiness across all frequency bands (Figure 9). The greatest increase in unsteadiness occurs at lower frequencies, and the greatest proportion of unsteadiness moves from $\mathrm{St} \in[0.4,0.6]$ for $\delta_{\mathrm{in}}=0.07 \mathrm{D}_{\text {in }}$ to $\mathrm{St} \in[0.2,0.4]$ for $\delta_{\mathrm{in}}=0.33 \mathrm{D}_{\text {in }}$ (Figure 9). Similar trends are observed for $\delta_{\text {in }}=0.51 D_{\text {in }}$ At higher frequencies, the contribution to the velocity fluctuations within the band which is close to the estimated one engine order $(\operatorname{St} \in[0.6,0.8])$ approximately doubles. Consequently there is an increased likelihood of an unsteady response of the compression system as the boundary layer thickness is increased. 
The effect of the flow profile asymmetry on the flow frequencies was also assessed. As the screen angle is increased to $\theta=45^{\circ}$ and $90^{\circ}$ for $\delta_{\mathrm{in}}=0.33 \mathrm{D}_{\mathrm{in}}$, there is a notable reduction in the flow unsteadiness, especially for the bands between $\mathrm{St}=0.0$ and $\mathrm{St}=0.6$ (Figure 10). This is in agreement with the spatial distributions of standard deviation in the velocity components (Figure 7) and is clearly beneficial in terms of overall flow distortion. However, it should be noted that in the range of frequencies of interest for aerodynamic response and aeromechanical forcing $(\mathrm{St}>0.7)$ the unsteadiness levels remain similar in magnitude to those for $\theta=0^{\circ}$. Therefore, a risk of unsteady response is of concern despite the reduction in unsteadiness for lower frequencies.

Overall, the results show that the thickness and orientation of the approaching total pressure profiles have a notable impact on the spectral content of the flow unsteadiness. As a consequence, a compression system at a particular operating point may be subjected to substantially different unsteady fluctuations for off-design airframe conditions compared to that at cruise where airframe incidence angles are expected to be small. Therefore, the design of distortion-tolerant fans must capture the wide range of possible spectral content in the unsteady swirl distortion to ensure that unsteady aerodynamic response and aeromechanical forcing are captured correctly.
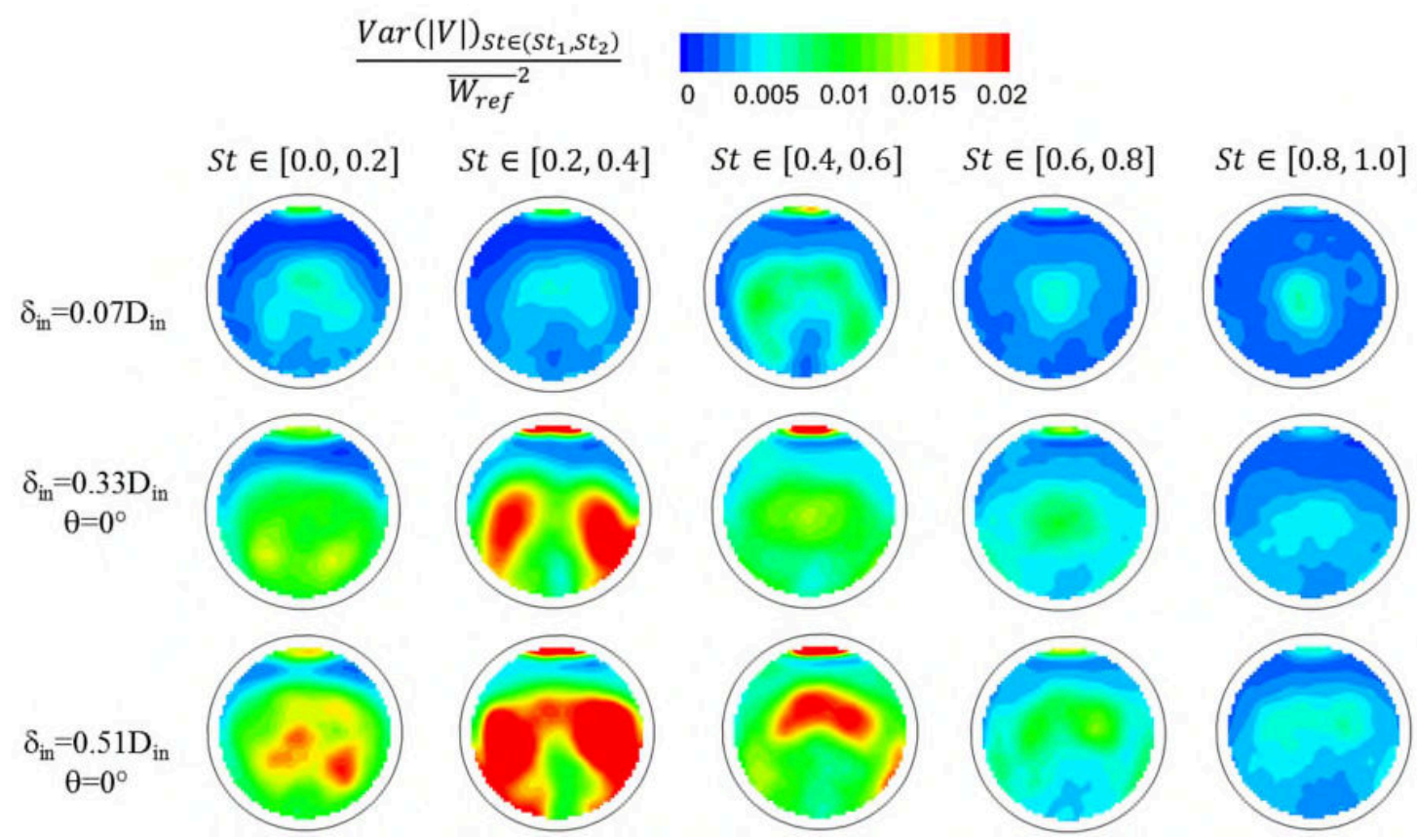

Figure 9: Spectral distribution of the velocity magnitude fluctuations for $\delta_{\text {in }}=0.07 \mathrm{D}_{\text {in }}, 0.33 \mathrm{D}_{\text {in }}$ and $0.51 \mathrm{D}_{\text {in }}$, $\left(\theta=\mathbf{0}^{\circ}\right)$ 


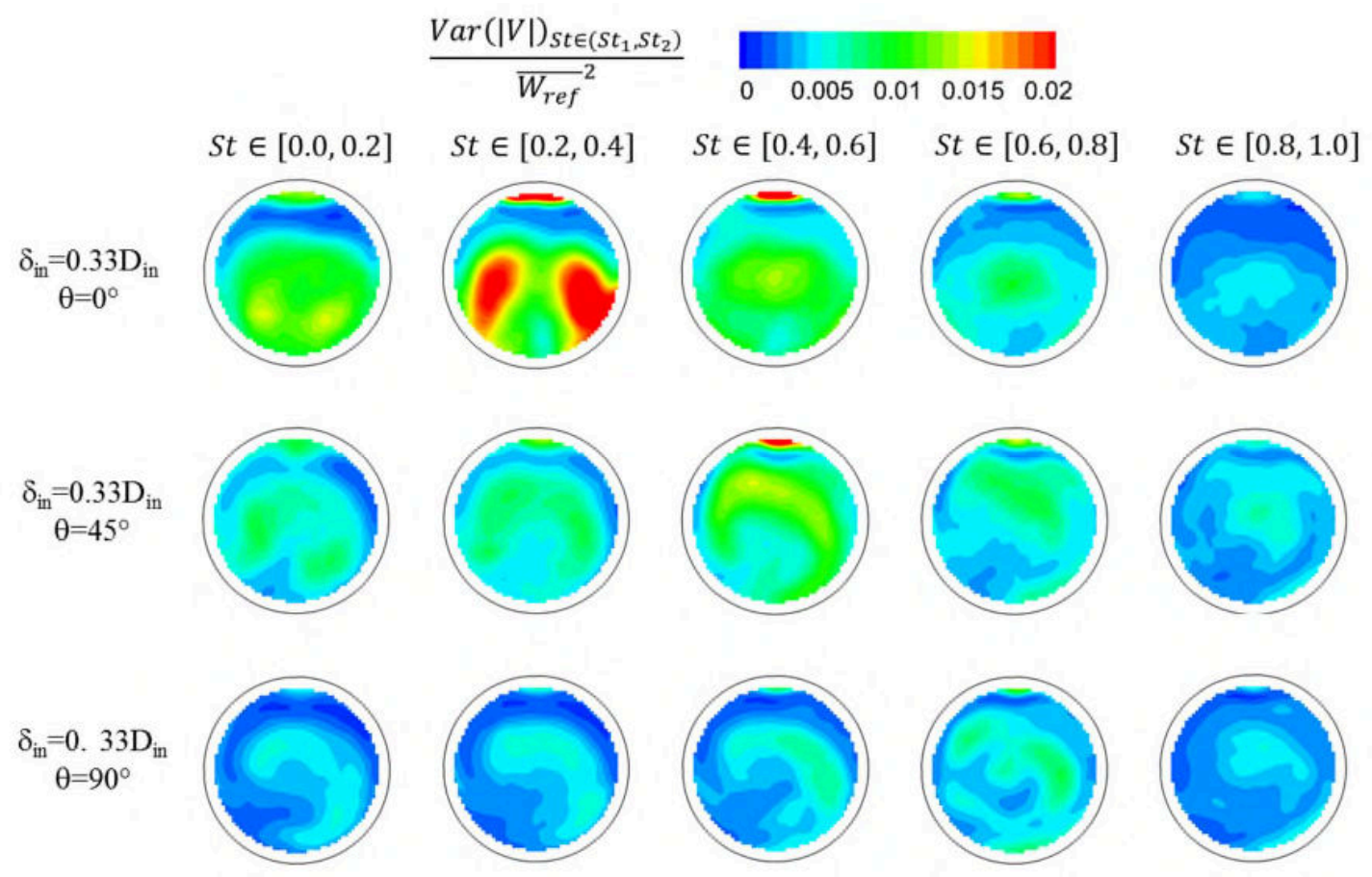

Figure 10: Spectral distribution of velocity magnitude normalised by reference velocity, $\delta_{\text {in }}=0.33 D_{\text {in }}, \theta=0^{\circ}$, $45^{\circ}, 90^{\circ}$

\section{Modal decomposition}

Proper Orthogonal Decomposition provides a means to identify key coherent structures which are responsible for most of the fluctuations in the flow field. The snapshot method [36] has been applied to the measurements in this study. The output of the analysis are the modes $\phi_{\mathrm{i}}(\mathrm{x}, \mathrm{y}), \mathrm{i}=1: \mathrm{N}_{\text {modes }}$ which represent the spatial distributions of each mode associated with the velocity fluctuations as evaluated for the complete time history of the measurements. It is then possible to reconstruct the flow field as a function of time by the summation of the modes scaled by the temporal coefficients $a_{i}(t)$. A useful property of this analysis is that the modes are ordered in terms of the relative contribution of each mode to the overall kinetic energy content in the velocity fluctuations. In this way, it is possible to identify the modes which contribute most to the unsteadiness in the flow field. Further details of the method used are reported by Gil-Prieto et al. [4].

Prior studies on S-duct flows [4][5] have demonstrated that two key modal structures exist. In one mode, termed the swirl switching mode, the in-plane velocity features a bulk swirl mode shape in which the flow oscillates between clockwise and counter-clockwise bulk swirl patterns. This is coupled with a lateral oscillation in the position of low axial-momentum region. This mode is associated with the Dean vortex motion which is widely reported downstream 
of bends in pipe flow [13][14]. The other key mode, termed the vertical mode [4], corresponds to a vertical oscillation in the position of the region of low axial momentum and is associated with the unsteady shear layer which originates from the flow separation upstream in the duct. The relative importance of the modes, and therefore the contribution to the overall kinetic energy content in the flow, depends on the offset ratio (H/L) of the S-duct [4]. For higher-offset Sducts such as that used in this study $(\mathrm{H} / \mathrm{L}=0.5)$, the swirl switching mode was found to be the most energetic mode (i.e. Mode 1), while the vertical mode was the second most energetic structure of the flow (i.e. Mode 2) [4]. In agreement with previous measurements, for the reference configuration $\left(\delta_{\text {in }}=0.07 \mathrm{D}_{\text {in }}\right)$ the swirl switching mode is the dominant mode (Figure 11). During this process there is a corresponding lateral oscillation in the position of the lowmomentum flow region (Figure 11). In the second mode, there is a modulation in the position and extent of the lowmomentum flow which oscillates between a topology where the secondary flows diminish, to one in which the region of loss is positioned close to the center of the AIP and the counter-rotating vortices re-emerge.

Both the swirl switching and vertical modes remain present when the inlet boundary layer thickness is increased to $\delta_{\text {in }}=0.33 \mathrm{D}_{\text {in }}$ and $\delta_{\text {in }}=0.51 D_{\text {in }}$ (Figure 11$)$. However, the vertical mode now contains the greatest level of overall kinetic energy which is indicated by the fact that this mode (Mode 1) appears prior to the lateral oscillation mode (Mode 2). The dominance of the vertical mode for thicker inlet boundary layers demonstrates that the separation in the S-duct is amplified by the ingestion of a thicker boundary layer on the lower surface of the S-duct inlet. This is because it is the boundary layer fluid on the lower half of the inlet plane to the duct which is mainly associated with the separation under the adverse pressure gradient after the first bend of the duct [11] . 

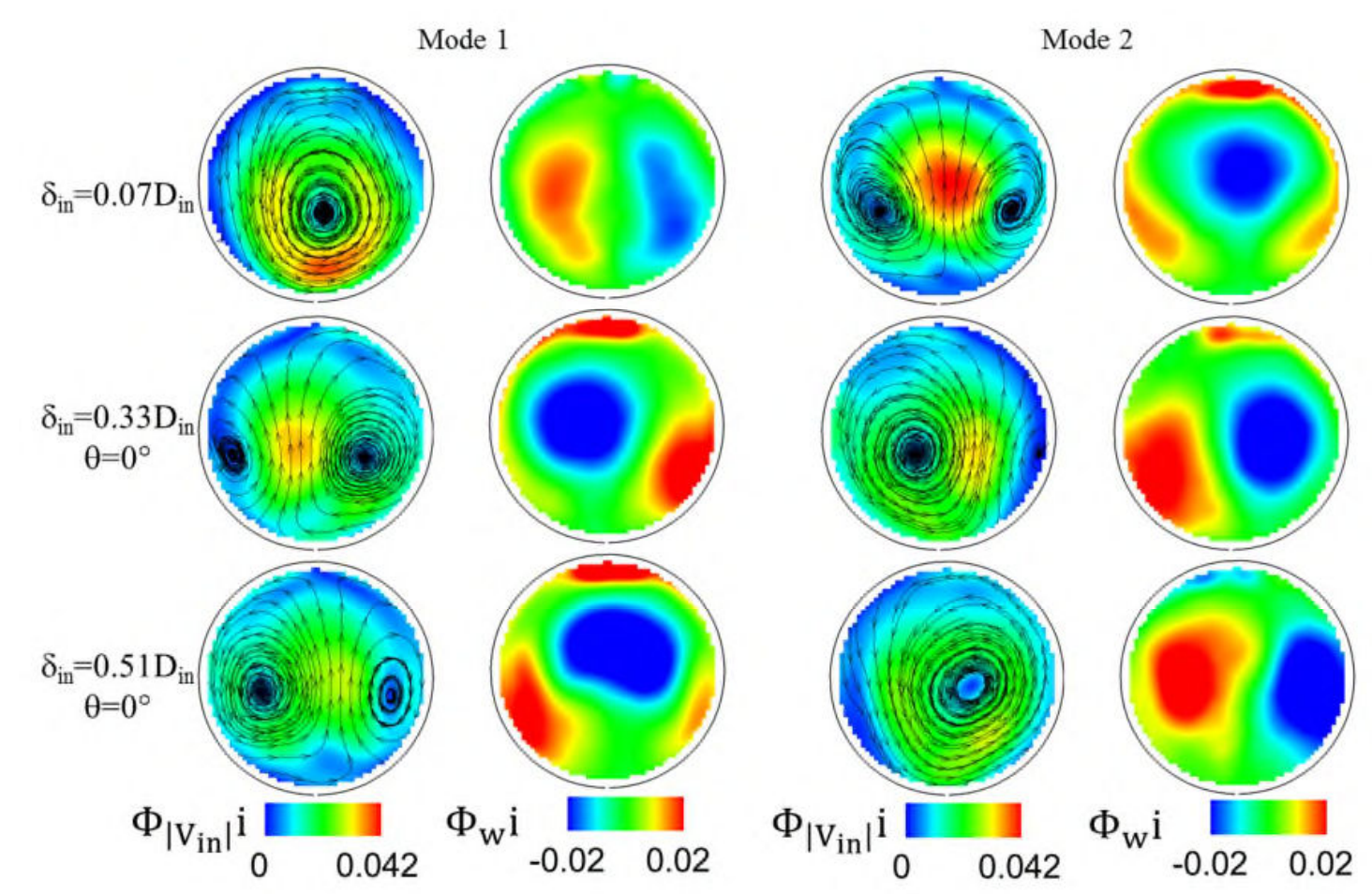

Figure 11: In plane and out of plane distributions of modes $i=1,2\left(\phi_{\text {Vin }}(i), \phi_{w}(i)\right)$, for $\delta_{\text {in }}=0.07 D_{\text {in }}, 0.33 D_{\text {in }}$ and 0.51D,$\left(\theta=0^{\circ}\right)$

The effect of the inlet flow profile asymmetry on the flow coherent structures was also assessed. The spatial distributions for the first two modes for $\theta=45^{\circ}$ are qualitatively similar to the vertical and swirl switching modes which characterize the $\theta=0^{\circ}$ case (Figure 12). However, the vertical mode becomes slightly tilted in a clockwise sense about the AIP centerline in agreement with the bias in the time-averaged secondary flows (Figure 6). The swirl switching mode still represents an alternating modulation in the strength of each vortex. However, the instantaneous flow is now biased in the clockwise direction. As a result, the flow oscillates between a clockwise bulk swirl and an offset paired swirl. Therefore, it is clear that the swirl switching mode no longer contains sufficient energy to fully reverse the flow to a counter-clockwise bulk swirl as was observed for a symmetric inlet boundary layer profile. The out of plane velocity follows the characteristic lateral oscillation in the position of the spoiled region, as observed in the $\theta=0^{\circ}$ cases.

Between $\theta=0^{\circ}$ and $\theta=90^{\circ}$ there is a gradual increase in tilt in the vertical mode distributions and bias in the clockwise direction for the swirl switching mode. At $\theta=90^{\circ}$ for $\delta_{\text {in }}=0.51 \mathrm{D}_{\text {in }}$ there is a significant change in the distributions for the first two modes (Figure 12). The most energetic mode comprises a mode shape which is characterized by a modulation in the size and position of the dominant vortex. The mode is associated with a 
modulation in the extent and position of the clockwise rotating cell, combined with a translation in the position of the main loss region. Mode 2 corresponds to a vertical type mode which shows that contributions to the flow's overall kinetic energy from shear layer oscillations remain important in the unsteadiness characteristics.

Overall, the analysis reveals that the characteristic swirl switching and vertical modes are broadly unchanged in spatial distribution for increased boundary layer thickness, although the vertical mode eventually becomes the dominant source of unsteadiness in the flow. The effect of the boundary layer asymmetry is to significantly change the spatial distributions of the characteristic S-duct modes. A consequence for engine-intake compatibility rig tests is that the key forcing functions for unsteady fan aerodynamic and structural response may not be captured correctly if appropriate levels of boundary layer thickness and degrees of asymmetry are not applied in the assessments.
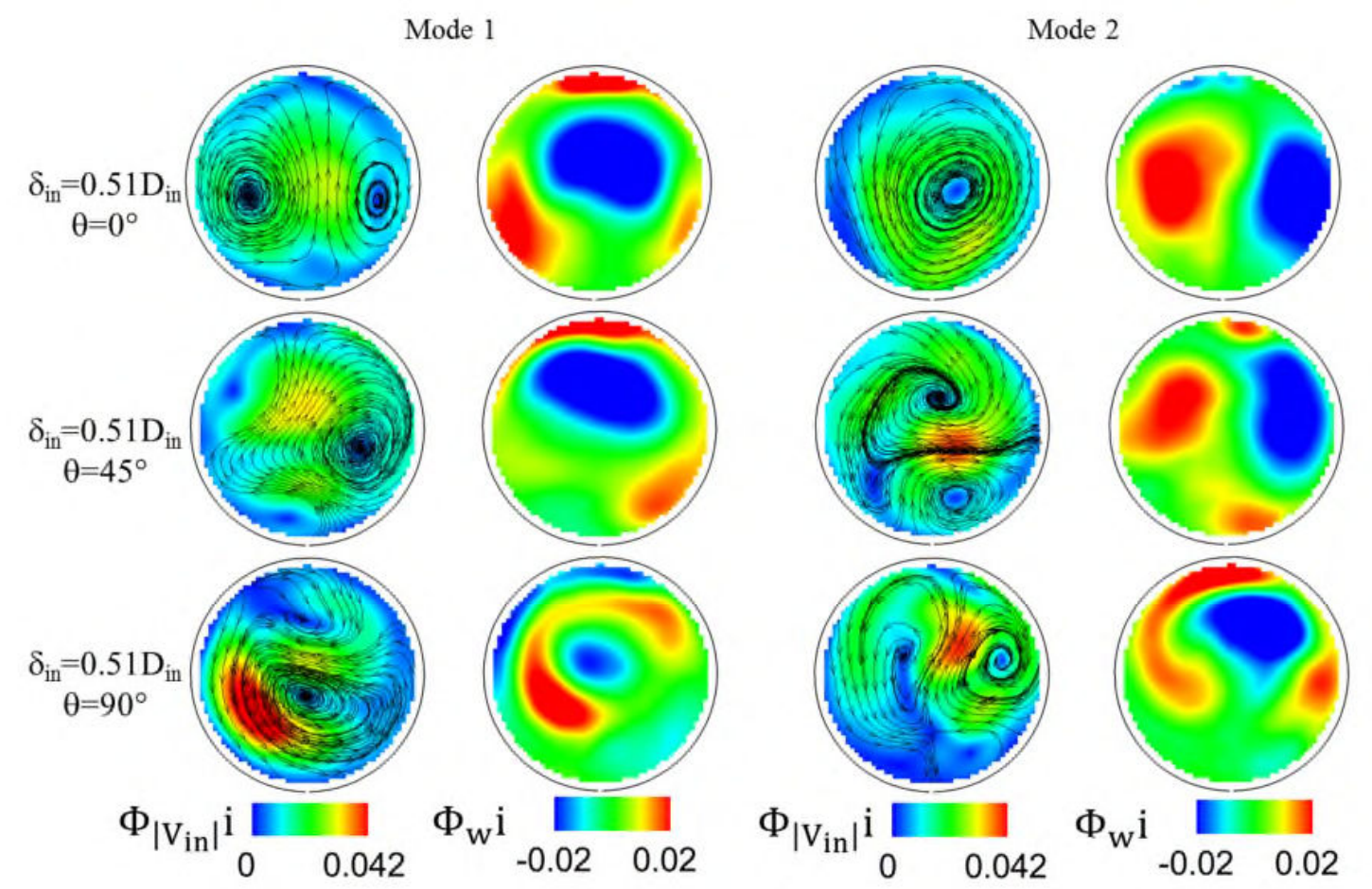

Figure 12: In plane and out of plane distributions for modes $i=1,2\left(\phi_{\text {Vin }}(i), \phi_{w}(i)\right), \delta_{\text {in }}=0.51 D_{\text {in }}$, for $\theta=0^{\circ}, 4^{\circ}$, $90^{\circ}$

\section{Distortion descriptors}

The swirl distortion descriptors [6] can be employed to characterize the levels of swirl distortion at the measurement plane. In this approach, the circumferential distribution of swirl angle is used to derive metrics termed the Swirl Intensity (SI), the Swirl Directivity (SD) and Swirl Pairs (SP) on equal area rings. The Swirl Intensity 
indicates the average absolute swirl angle on the ring. The Swirl Directivity provides a measure of the overall direction of rotation of the flow. For example, a value of SD of 1.0 corresponds to a flow with a bulk rotation in the counterclockwise sense and a twin-swirl pattern features an SD of 0.0. The Swirl Pairs (SP) parameter quantifies the number of pairs of swirl with alternating direction over a circumferential distribution. A bulk swirl topology corresponds to $\mathrm{SP}=0.5$. Details of the formulation of SI, SP and SD can be obtained in [9]. Linear interpolation has been employed to interpolate the swirl angle measurements on to a rake resolution of $5^{\circ}$ in the circumferential direction and using the four inner-most radial positions of $\mathrm{r} / \mathrm{R}=[0.316,0.548,0.707,0.837]$ associated with the typical five equal-area rings considered in industry [6]. The outer-most radial position $r / R=0.949$ has not been considered in this analysis as $r / R$ $=0.95$ represents the edge of the reliable PIV data. The resolution of $5^{\circ}$ exceeds the number of rakes of the industry practice, where the resolution is usually $45^{\circ}$, and this was found to increase the SI by up to $8 \%$.

For the baseline case $\left(\delta_{\text {in }}=0.07 \mathrm{D}_{\text {in }}\right)$, the flow features the widely-reported swirl switching mechanism as observed in previous studies on the same S-duct geometry [3][4][5] and in the modal decomposition (Figure 11). This mode results in high levels of probability at $\mathrm{SP}=0.5$ and $\mathrm{SD}=+/-1.0$ (Figure 13), which correspond to a dominant bulk swirl topology, as well as an intermediate state at $\mathrm{SP}=1.0, \mathrm{SD}=0.0$ which corresponds to a twin swirl pattern. The SP-SD distributions remain broadly consistent across all radial positions, even though the bulk swirl topology becomes less dominant towards the tip region. The peak swirl intensity events are associated with the bulk swirl events at $\mathrm{SP}=0.5$ (Figure 14) which is in agreement with previous studies. The maximum SI at the tip region was found in the order of $15.0^{\circ}$. The swirl distortion levels are greatest however at the hub region where a maximum SI of $30.2^{\circ}$ was observed

When the thickness of the boundary layer is increased from $0.07 \mathrm{D}_{\text {in }}$ to $0.51 \mathrm{D}_{\text {in }}$ there is an increase in the PDF associated with $\mathrm{SP}=1.0, \mathrm{SD}=0$ which reflects a greater tendency towards twin swirl patterns (Figure 13). This result is consistent with the proper orthogonal decomposition analysis which revealed that the vertical mode becomes dominant as in the POD mode classification it appears as Mode 1, indicating the highest level of overall kinetic energy (Figure 11). There is also a significant increase in the swirl intensity levels with greater values of $\delta_{\text {in }}$ (Figure 14). The highest overall levels remain in the hub region. For example, for $\delta_{\text {in }}=0.51 \mathrm{D}_{\text {in }}$, the peak values reach $\mathrm{SI}=40.3^{\circ}($ Figure 14). A similar increase in swirl distortion levels as $\delta_{\text {in }}$ increases is observed in the tip region (Figure 14). The maximum value of SI increases by $40 \%$ to $21.0^{\circ}$ for $\delta_{\text {in }}=0.51 \mathrm{D}_{\text {in }}$, relative to the baseline flow profile case. Overall, the amplification of the swirl distortion levels when $\delta_{\text {in }}$ increases underlines the importance of the use of appropriate profiles for the approaching boundary layer for engine-intake compatibility studies. 
The effect of the inlet flow profile asymmetry on the swirl descriptors maps was also evaluated. The time-averaged swirl angle distributions (Figure 5 and Figure 6) revealed that the average flow field at the AIP progressively moves to a dominant swirl direction in the lower half of the AIP as the asymmetry of the approaching boundary layer $(\theta)$ is increased. This topology promotes dominant swirl in the anti-clockwise direction at the hub region of the measurement plane, as indicated by a bias in the $\mathrm{SP}-\mathrm{SD}$ probability distributions towards $\mathrm{SD}=1.0$ and $\mathrm{SP}=0.5$ for $\theta=90^{\circ}$ (Figure 15 ) which becomes very notable at the tip region when $\delta_{\text {in }}=0.51 D_{\text {in }}$. At $\delta_{\text {in }}=0.51 D_{\text {in }}$ the bias is sufficiently strong such that only clockwise swirl is observed. The flow topology at the hub was found highly unsteady in a manner which is similar to the symmetric boundary layer cases $\left(\theta=0^{\circ}\right)$ as shown by the persistence of negative swirl $(\mathrm{SD}=-1.0)$ and twin swirl $(\mathrm{SD}=0.5, \mathrm{SP}=1.0)$ patterns. A key effect of inlet boundary layer asymmetry, therefore, is to polarize the swirl directivity between the hub and the tip.

Despite the significant changes in the flow topology, the peak swirl events for asymmetric boundary layers remain associated with bulk-swirl topologies across the AIP in both the hub and tip regions (Figure 16). At the tip position the mean swirl intensity as a function of $\theta$ is strongly dependent on $\delta_{\text {in }}$. This is more notable for the $\delta_{\text {in }}=0.51 D_{\text {in }}$ case where the mean SI exceeds $15.0^{\circ}$ for $\theta=90^{\circ}$ from around $10^{\circ}$ for $\theta=0^{\circ}$. The symmetry of the inlet profile had a weak impact on the mean SI for the $\delta_{\text {in }}=0.33 \mathrm{D}_{\text {in }}$ case (see Figure 17) as a small variation of the man SI was observed between the $\theta=0^{\circ}$ and $\theta=90^{\circ}$ cases. In terms of the SI peak values, for $\delta_{\text {in }}=0.33 \mathrm{D}_{\text {in }}$ there is a slight reduction in peak SI values from $20.0^{\circ}$ to $17.0^{\circ}$ as $\theta$ increases from 0 to $90^{\circ}$ (Figure 17 ). However, for $\delta_{\text {in }}=0.51 \mathrm{D}_{\text {in }}$, there is a notable rise in swirl distortion levels with $\theta$ (Figure 16 and Figure 17). At $\theta=90^{\circ}$, the maximum value of SI increases from $21.0^{\circ}$ to $27.1^{\circ}$. This rise is predominantly driven by the increase in the average value of SI due to the strong clockwise bias which is found in the tip regions of the flow field (Figure 6). This is an important result from the perspective of the fan response as flow distortion in the tip region is likely to have a strong influence on overall fan stability[21][22]. 

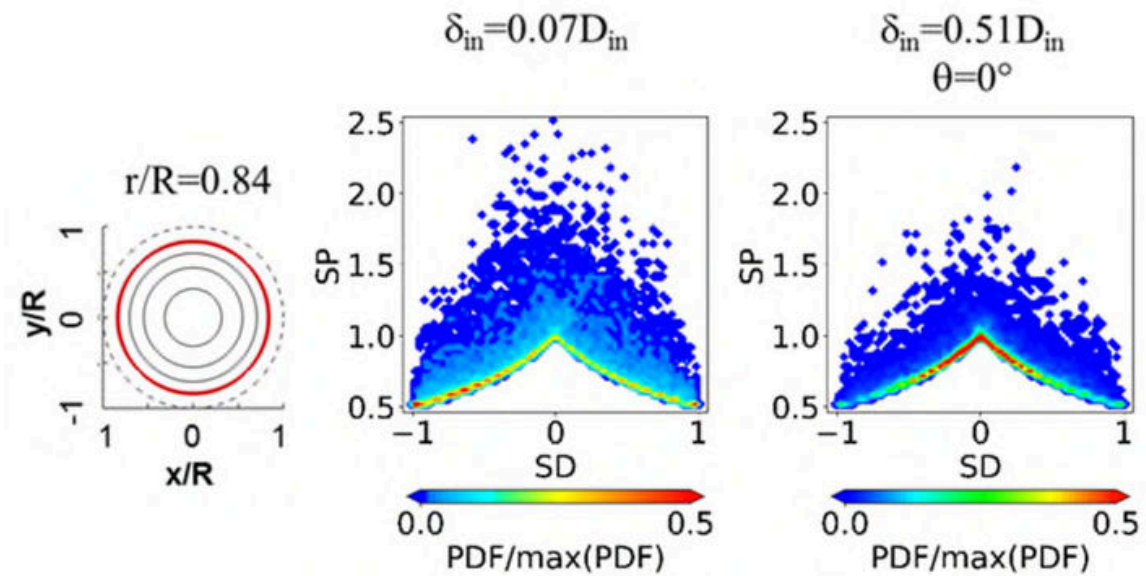

Figure 13: Normalised Probability Density Functions (PDF*) based on SP and SD for the tip region $(\mathbf{r} / \mathbf{R}=\mathbf{0 . 8 4})$, for $\delta_{\text {in }}=0.07 D_{\text {in }}$ and $0.51 D_{\text {in }}$ with $\theta=0^{\circ}$.
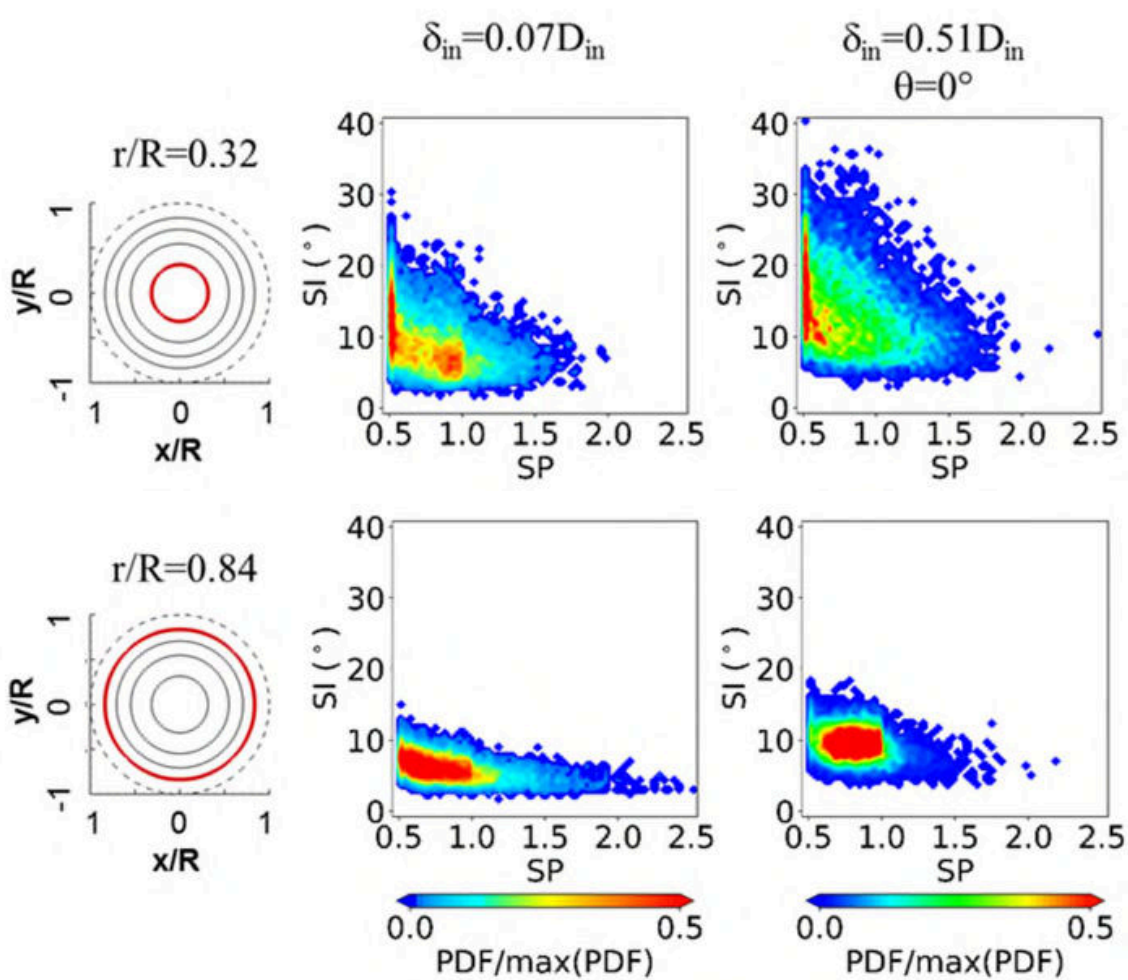

Figure 14: Normalised Probability Density Functions (PDF*) of SP and SI for hub $(r / R=0.32)$ and tip $(\mathrm{r} / \mathrm{R}=\mathbf{0 . 8 4}), \delta_{\mathrm{in}}=\mathbf{0 . 0 7}_{\mathrm{in}}$ and $0.51 \mathrm{D}_{\text {in }}$ with $\theta=0^{\circ}$. 

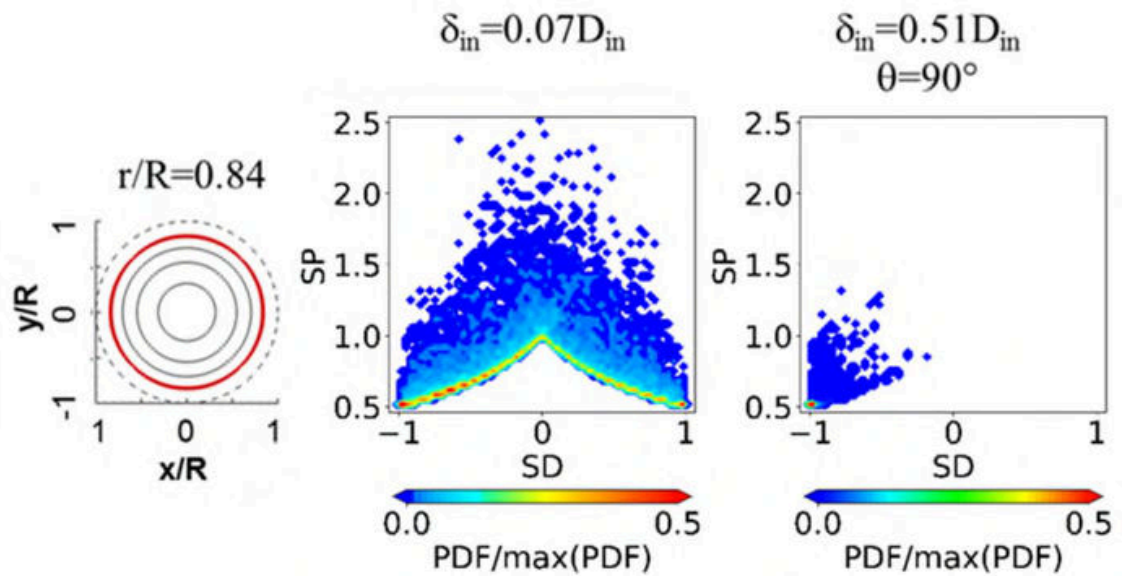

Figure 15: Normalised Probability Density Functions (PDF*) of SP and SD for the tip region $(\mathbf{r} / \mathbf{R}=0.84), \delta_{\text {in }}=0.07 D_{\text {in }}$ and $0.51 D_{\text {in }}$ with $\theta=0^{\circ}$ and $90^{\circ}$.
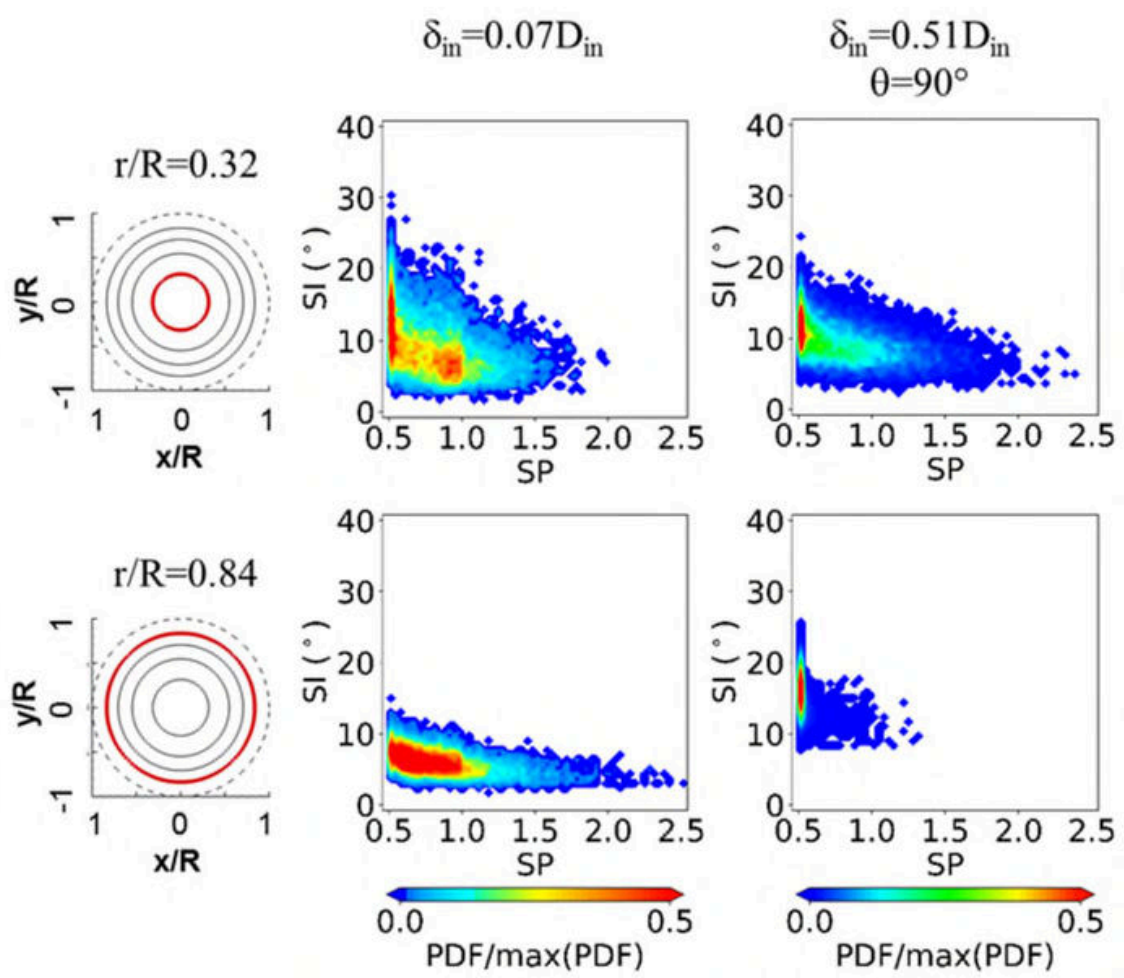

Figure 16: Normalised Probability Density Functions (PDF*) of SP and SI for hub $(\mathbf{r} / \mathbf{R}=0.32)$ and tip $(r / R=0.84)$ rings, $\delta_{\text {in }}=0.07 D_{\text {in }}$ and $0.51 D_{\text {in }}$ with $\theta=0^{\circ}$ and $90^{\circ}$. 


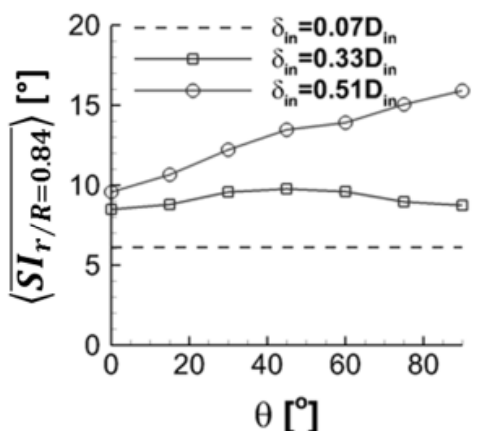

(a)

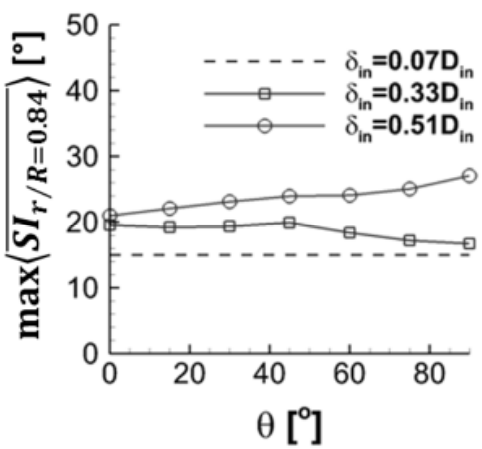

(b)

Figure 17: Mean (a) and peak (b) swirl intensity (SI) as a function of screen angle $(\theta)$ for tip position $r / R=0.84$ 


\section{IV.Conclusions}

A detailed experimental survey has been conducted to determine the effect of the inlet flow profile on the intake flow distortion at the outlet of an S-duct intake. Three-component velocity measurements of high spatial and temporal resolution were acquired using time resolved PIV. Mesh-based distortion screens were mounted upstream of the Sduct inlet plane to generate total pressure profiles which are expected to be representative of possible approaching boundary layers on boundary layer ingestion airframe configurations.

An increase in the thickness of the approaching boundary layer promotes an intensification of the classic vortical structures at the downstream exit of the S-duct. Modal decomposition reveals that the characteristic unsteady modes for S-ducts, namely the swirl switching and shear layer oscillations, are not fundamentally altered. However, the increase in boundary layer thickness results in additional flow unsteadiness in the shear layer, most likely due to a deepening of the flow separation upstream inside the duct due to the adverse static pressure gradients. The spectral content of the flow field fluctuations reveals that for an increased boundary layer thickness, peak flow unsteadiness occurs at frequencies which are approximately half of those which are found on the baseline configuration. However, there remains notable unsteadiness at frequencies which are most pertinent for unsteady aerodynamic and aeromechanical response in the compression system. The consequence of the increase in average and unsteady swirl angle is that the peak swirl distortion, expressed in terms of Swirl Intensity, increases by up to $40 \%$ when the boundary layer thickness increases from $\delta_{\text {in }} / D_{\text {in }}=0.07$ to 0.51 .

The measurements also confirm that the asymmetry of the approaching boundary layer relative to the intake centerline has a notable impact on the swirl distortion at the AIP. As the level of asymmetry is increased, the timeaveraged flow field tends towards a topology with a dominant vortex. There is a notable reduction in overall flow field unsteadiness at the AIP, although flow unsteadiness remains present at frequencies which are of note for turbomachinery response. Strong radial gradients in swirl distortion are introduced such that the peak levels of swirl intensity reduce in the regions close to the hub, but increase by up to $29 \%$ in tip regions due to an increase in the average swirl intensity, if the flow profile is rotated by 90 degrees relative to the symmetric profile.

For novel aircraft architectures, this highlights the importance of assessing the aerodynamics of propulsion integration under a range of representative conditions. Appropriate levels of boundary layer thickness and asymmetry 
must be considered as part of intake-engine compatibility assessments to ensure that the spatial and temporal characteristics of the intake flow are well-represented.

\section{Funding Sources}

This work was co-funded by Innovate UK.

\section{References}

[1] Liebeck, R. H., "Design of the Blended Wing Body Subsonic Transport," Journal of Aircraft, Vol. 41, No. 1, 2004, pp. 10-25, doi: $10.2514 / 1.9084$

[2] Garnier, E., "Flow Control by Pulsed Jet in a Curved S-Duct: A Spectral Analysis," AIAA Journal, Vol. 53, No. 10, 2015, pp. 2813-2827. doi: 10.2514/1.J053422

[3] Zachos, P. K., MacManus, D. G., Gil-Prieto, D., and Chiereghin, N., "Flow Distortion Measurements in Convoluted Aeroengine Intakes," AIAA Journal, Vol 54, No. 9, 2016, pp. 2819-2832, doi: 10.2514/1.J054904.

[4] Gil-Prieto, D., MacManus, D. G., Zachos, P. K., Tanguy, G., and Menzies, K. R., "Convoluted Intake Distortion Measurements Using Stereo Particle Image Velocimetry,” AIAA Journal, Vol 55, No 6, 2017, pp. 1878-1892, doi: 10.2514/1.J055467.

[5] Gil-Prieto, D., Macmanus, D. G., Zachos, P. K., Tanguy, G., Wilson, F., and Chiereghin, N., "Delayed DetachedEddy Simulation and Particle Image Velocimetry Investigation of S-Duct Flow Distortion,” AIAA Journal, Vol. 55, No. 6, 2017, doi: 10.2514/1.J055468

[6] Society of Automotive Engineers, “A Methodology for Assessing Inlet Swirl Distortion," SAE Aerospace Information Report AIR5686, Warrendale, PA, USA, 2007.

[7] Florea, R. V., Matalanis, C., Hardin, L. W., Stucky, M., and Shabbir, A., "Parametric Analysis and Design for Embedded Engine Inlets," Journal of Propulsion and Power, Vol. 31, No. 3, 2015, pp. 843-850, doi: 10.2514/1.B34804.

[8] Provenza, A.J., Duffy, K.P., and Bakhle, M.A., “Aeromechanical Response of a Distortion-Tolerant Boundary Layer Ingesting Fan,” J. Eng. Gas Turbines Power, Vol. 141, No. 1, 2018, doi: 10.1115/1.4040739

[9] Owens, L. R., Allan, B. G., and Gorton, S. A., "Boundary-Layer-Ingesting Inlet Flow Control,” Journal of Aircraft, Vol. 45, No. 4, 2008, pp. 1431-1440, doi: 10.2514/1.36989.

[10] Tanguy, G., MacManus, D. G., Zachos, P., Gil-Prieto, D., and Garnier, E., "Passive Flow Control Study in an S-Duct Using Stereo Particle Image Velocimetry," AIAA Journal, Vol. 55, No. 6, 2017, pp. 1862-1877, doi: 10.2514/1.J055354. 
[11] Wellborn, S. R., Reichert, B. A., and Okiishi, T. H., "Study of the Compressible Flow in a Diffusing S-Duct," Journal of Propulsion and Power, Vol. 10, No. 5, 1994, pp. 668-675, doi: 10.2514/3.23778.

[12] Gil-Prieto, D. ., Zachos, P. K. ., MacManus, D. G., and McLelland, G., "Unsteady Characteristics of S-Duct Intake Flow Distortion,” Aerospace Science and Technology, Vol. 84, 2019, pp. 938-952, doi: 10.1016/j.ast.2018.10.020.

[13] Hellström, L. H. O., Zlatinov, M. B., Cao, G., and Smits, A. J., "Turbulent Pipe Flow Downstream of a 90 Bend," Journal of Fluid Mechanics, Vol. 735, R7, 2013, doi: 10.1017/jfm.2013.534.

[14] Kalpakli Vester, A., Örlü, R., and Alfredsson, P. H., "POD Analysis of the Turbulent Flow Downstream a Mild and Sharp Bend," Experiments in Fluids, Vol. 56, No. 3, 2015, doi: 10.1007/s00348-015-1926-6.

[15] Giuliani, J. E., and Chen, J.-P., "Fan Response to Boundary-Layer Ingesting Inlet Distortions," AIAA Journal, Vol. 54, No. 10, 2016, pp. 3232-3243, doi: 10.2514/1.J054762.

[16] Rein, M., and Koch, S., "Experimental Study of Boundary-Layer Ingestion into a Diverterless S-Duct Intake," AIAA Journal, Vol. 53, No. 11, 2015, pp. 3487-3491, doi: 10.2514/1.J053902.

[17] Kim, H., and Liou, M.-S., "Flow Simulation of N2B Hybrid Wing Body Configuration," 50th AIAA Aerospace Sciences Meeting Including the New Horizons Forum and Aerospace Exposition, AIAA 2012-0838, Nashville, Tennesee, USA, 9-12 January 2012, doi: 10.2514/6.2012-838.

[18] Berens, T. M., Delot, A.-L., Tormalm, M. H., Ruiz-Calavera, L. P., Funes-Sebastian, D. E., Rein, M., Säterskog, M., Ceresola, N., and Zurawski, L., "Numerical and Experimental Investigations on Highly Integrated Subsonic Air Intakes," 52nd Aerospace Sciences Meeting, AIAA, 2014, doi: 10.2514/6.2014-0722

[19] Guimarães, T., Lowe, K.T., and O’Brien, W.F., “Complex Flow Generation and Development in a Full-Scale Turbofan Inlet,” J. Eng. Gas Turbines and Power, Vol. 140, No. 8, 2018, doi: 10.1115/1.4039179

[20] Gunn, E. J., Tooze, S. E., Hall, C. A., and Colin, Y., “An Experimental Study of Loss Sources in a Fan Operating With Continuous Inlet Stagnation Pressure Distortion,” Journal of Turbomachinery, Vol. 135, No. 5, 2013, pp. 51002-51010, doi: 10.1115/1.4007835.

[21] Cousins, W. T., "History, Philosophy, Physics, and Future Directions of Aircraft Propulsion System/Inlet Integration," Proceedings of ASME Turbo Expo 2004 Power for Land, Sea, and Air, GT2004-54210, Vienna, Austria, 14-17 June 2004, doi: 10.1115/GT2004-54210.

[22] Page, J., Hield, P., and Tucker, P., "Effect of Inlet Distortion Features on Transonic Fan Rotor Stall," Journal of Turbomachinery, Vol. 140, No. 7, 2018, pp. 71008-71011, doi: 10.1115/1.4040030.

[23] Meyer, W., Pazur, W., and Fottner, L., "The Influence of Intake Swirl Distortion on the Steady-State Performance of a Low Bypass, Twin-Spool Engine," AGARD CP-498, September 1992. 
[24] Lee, K.-B., Wilson, M., and Vahdati, M., "Effects of Inlet Disturbances on Fan Stability,” J. Eng. Gas Turbines and Power, Vol. 141, No. 5, 2018, doi: 10.1115/1.4042204

[25] Vahdati. M., and Cumpsty, N., “Aeroelastic Instability in Transonic Fans,” J. Eng. Gas Turbines and Power, Vol. 138, No. 2, 2015, doi: 10.1115/1.4031225

[26] Wieneke, B., "Stereo-PIV Using Self-Calibration on Particle Images," Experiments in Fluids, Vol. 39, No. 2, 2005, pp. 267-280, doi: 10.1007/s00348-005-0962-z.

[27] Raffel, M., Willert, C. E., Wereley, S. T., and Kompenhans, J., Particle Image Velocimetry: A Practical Guide, $2^{\text {nd }}$ Ed, Springer-Verlag Berlin, Heidelberg, 2007, doi: 10.1007/978-3-540-72308-0.

[28] Birch, D., Martin, N., "Tracer particle momentum effects in vortex flows," Journal of Fluid Mechanics, vol. 723, pp. 665-691, 2013, doi: 10.1017/jfm.2013.82.

[29] Zhou, M. "Particle Image Velocimetry Applied to Non-reacting and Reacting Flows within Cylindrical Combustion Chambers”, Ph. D. dissertation, Chapter 3, p.66, Loughborough University, UK, 1996.

[30] Berrier, B. L., Carter, M. B., Allan, B. G., "High Reynolds Number Investigation of a Flush-Mounted, S-Duct Inlet With Large Amounts of Boundary Layer Ingestion”, NASA Technical Paper TP-2005-213766, Langley Research Centre, Hampton, Virginia, 2005.

[31] Gunn, E. J., Hall, C. A., “Aerodynamics of Boundary Layer Ingesting Fans,” Proceedings of ASME Turbo Expo, 1620 June 2014, Dusseldorf, Germany, GT2014-26142, doi: 10.1115/GT2014-26142

[32] Breuer, T., and Bissinger, N. C., "Basic Principles: Gas Turbine Compatibility - Gas Turbine Aspects,” Encyclopedia of Aerospace Engineering, edited by R. Blockley and W. Shyy, 2010, doi: 10.1002/9780470686652.eae573.

[33] Younghans, J. L., and Paul, D. L., “Considerations in Inlet/Engine Integration,” The Aerothermodynamics of Aircraft Gas Turbine Engines, AFAPL-TR-78-52, Air Force Aero Propulsion Laboratory, Ohio, USA, 1978.

[34] Strazisar, A. J., Wood, J. R., Hathaway, M. D., and Suder, K. L., "Laser Anemometer Measurements in a Transonic Axial-Flow Fan Rotor", NASA TP-2897, November 1989.

[35] Welch, P. D., "The Use of Fast Fourier Transform for the Estimation of Power Spectra: A Method Based on Time Averaging Over Short, Modified Periodograms," IEEE Transactions on Audio and Electroacoustics, Vol. 15, No. 2 , 1967, pp. 70-73, doi: 10.1109/TAU.1967.1161901.

[36] Sirovich, L., "Turbulence and the Dynamics of Coherent Structures. I. Coherent Structures," Quarterly of Applied Mathematics, Vol. 45, No. 3, 1987, pp. 561-571, doi: https://doi.org/10.1090/qam/910462 
2020-04-15

\section{Influence of upstream total pressure profiles on S-duct intake flow distortion}

McLelland, Grant

AIAA

McLelland G, MacManus DG, Zachos PK,et al., (2020) Influence of upstream total pressure profiles on S-duct intake flow distortion. Journal of Propulsion and Power, Volume 36, Issue 3, May 2020, pp.346-356

https://doi.org/10.2514/1.B37554

Downloaded from Cranfield Library Services E-Repository 